\title{
Diffusion of a passive scalar from a no-slip boundary into a two-dimensional chaotic advection field
}

\author{
By S. GHOSH$H^{1} \dagger$, A. LEONARD ${ }^{2}$ AND S. WIGGINS \\ ${ }^{1}$ Chemical Engineering, California Institute of Technology, Pasadena, CA 91125, USA \\ ${ }^{2}$ Graduate Aeronautical Laboratories, California Institute of Technology, Pasadena, CA 91125, USA \\ ${ }^{3}$ Applied Mechanics, California Institute of Technology, Pasadena, CA 91125, USA
}

(Received 10 June 1994 and in revised form 12 May 1998)

Using a time-periodic perturbation of a two-dimensional steady separation bubble on a plane no-slip boundary to generate chaotic particle trajectories in a localized region of an unbounded boundary layer flow, we study the impact of various geometrical structures that arise naturally in chaotic advection fields on the transport of a passive scalar from a local 'hot spot' on the no-slip boundary. We consider here the full advection-diffusion problem, though attention is restricted to the case of small scalar diffusion, or large Péclet number. In this regime, a certain one-dimensional unstable manifold is shown to be the dominant organizing structure in the distribution of the passive scalar. In general, it is found that the chaotic structures in the flow strongly influence the scalar distribution while, in contrast, the flux of passive scalar from the localized active no-slip surface is, to dominant order, independent of the overlying chaotic advection. Increasing the intensity of the chaotic advection by perturbing the velocity field further away from integrability results in more non-uniform scalar distributions, unlike the case in bounded flows where the chaotic advection leads to rapid homogenization of diffusive tracer. In the region of chaotic particle motion the scalar distribution attains an asymptotic state which is time-periodic, with the period the same as that of the time-dependent advection field. Some of these results are understood by using the shadowing property from dynamical systems theory. The shadowing property allows us to relate the advection-diffusion solution at large Péclet numbers to a fictitious zero-diffusivity or frozen-field solution, corresponding to infinitely large Péclet number. The zero-diffusivity solution is an unphysical quantity, but is found to be a powerful heuristic tool in understanding the role of small scalar diffusion. A novel feature in this problem is that the chaotic advection field is adjacent to a no-slip boundary. The interaction between the necessarily non-hyperbolic particle dynamics in a thin near-wall region and the strongly hyperbolic dynamics in the overlying chaotic advection field is found to have important consequences on the scalar distribution; that this is indeed the case is shown using shadowing. Comparisons are made throughout with the flux and the distributions of the passive scalar for the advection-diffusion problem corresponding to the steady, unperturbed, integrable advection field.

$\dagger$ Present address: ARCO Exploration and Production Technology, Plano, TX 75075 USA. 


\section{Introduction}

The kinematics of a perfect or non-diffusive tracer is purely advective. In other words, perfect tracer particles will follow the pathlines of the flow. The dispersion of these particles is then directly related to the fluid particle trajectories and, in Eckart's terminology (see Eckart 1948), is called 'stirring. Given some initial distribution of perfect tracer particles, how this distribution evolves in time is entirely dependent on the dynamics of particle motion in the flow, and therefore the associated transport issues are often best understood using the global geometrical viewpoint of dynamical systems theory. In stirring by chaotic advection (Aref 1984), the individual particle trajectories might be very complicated, but the underlying geometrical structures such as invariant manifolds and homoclinic/heteroclinic tangles provide a dynamical template that in certain cases considerably simplifies questions related to the transport or dispersion of particles (e.g. see Wiggins 1992). A more realistic scalar impurity will, however, undergo both advection and diffusion. Thus the time-evolution of some given initial scalar field will be dictated not only by the purely fluid-mechanical stirring process but also by the generally slower process of molecular diffusion of the now diffusive tracer, which is called 'mixing' (Eckart 1948). In the Lagrangian framework, the kinematics of a diffusive tracer has a Brownian-motion component in addition to the advective component due to the fluid motion, and tracer particles no longer follow the pathlines of the flow. That raises several fundamental questions regarding the role of the underlying geometrical structures in the transport of a passive scalar and the manner in which they influence the time-evolution of a scalar field, particularly at small scalar diffusivities. Moreover, scalar advection in chaotic flows creates finescale structure since the attendant strong stretching and folding operations result in arbitrarily small striation thicknesses (Aref \& Jones 1989; Jones 1991), so that even asymptotically small diffusivity cannot be ignored. It is therefore also a matter of considerable practical importance to incorporate small scalar diffusion. Among other considerations is the relationship between the stirring and mixing processes, in particular how small scalar diffusion affects the zero-diffusivity solution corresponding to pure advection.

An important isssue which has been mostly ignored in the existing literature is the transport of a passive scalar from an active no-slip boundary into a chaotic advection field, even though heat and mass transfer from stationary surfaces is common in engineering applications. A stagnation point (or fixed point) is called hyperbolic if the velocity field expanded about the stagnation point has no eigen-values with zero real part. The linear part of the velocity field expanded about any stagnation point on the no-slip boundary has zero eigen-values, and therefore every point on the no-slip boundary is non-hyperbolic. The non-hyperbolicity of the stagnation points on a noslip boundary makes analysis difficult. Further, stirring, by itself, becomes meaningless since diffusion is essential for 'lifting' heat or a passive impurity from the active noslip surface. Given these complications, it is not clear how the geometrical structures in a chaotic advection field adjacent to an active no-slip surface can influence the time-dependent distribution of the scalar field as the scalar impurity diffuses into the flow. Our objective is to investigate some of these issues using a simple twodimensional time-periodic separation bubble with chaotic particle trajectories, over a plane stationary surface.

We use a method devised by Perry \& Chong (1986a) to obtain a simple Taylorseries representation of a chaotic separation bubble which is also an asymptotically exact solution of the Navier-Stokes and continuity equations, close to the origin of the series-expansion. The method relies on the availability of a sufficient number of topological constraints (Perry \& Chong 1986a) and is therefore particularly wellsuited to study steady two- and three-dimensional separated flows (Perry \& Chong 
$1986 a$; Tobak \& Peake 1982; Dallmann 1988) on account of their readily available topological features such as location and stability-type of stagnation points, location of points of zero shear stress on the no-slip boundary, angles of separation and attachment, etc. Our scheme is to construct a low-order series-representation of a steady two-dimensional separation bubble at a plane wall and then introduce time-periodic terms to obtain an unsteady bubble with chaotic particle trajectories, such that the representation satisfies incompressibility and remains an asymptotically exact solution of the now time-dependent Navier-Stokes equations. The truncated series-solution constitutes a simple time-periodic perturbation of an integrable dynamical system. There are methods (Perry \& Chong 1986a) for testing the accuracy of a truncated series-solution over any given region of the flow, but we will not concern ourselves with identifying a domain of applicability of the series-solution since attention will be mostly confined to regions close to the origin of the expansion. Only a localized portion of the plane wall is considered as an active surface such that finite-time distributions of the scalar field remain confined near the origin of the series-expansion.

The relative importance of advection versus diffusion is measured by the Péclet number, which is the ratio of the diffusion and advection time-scales. We are interested in the regime of small scalar diffusion, or more precisely, the regime where the diffusion time-scale is much greater than the advection time-scale, which means large Péclet numbers. At large Péclet numbers, the scalar advection-diffusion problem is best tackled by random-walk methods based on the theory of Brownian motion (Wang \& Uhlenbeck 1945), and we develop numerical implementations of these methods to solve for the time-dependent scalar field. These computations show clearly that for scalar transport with small scalar diffusivity, the unstable manifold of a saddle point on the boundary corresponding to zero shear stress is the organizing structure in the distribution of the scalar field, and the time evolution of the scalar field can be understood in terms of the structure of the unstable manifold. This is a central theme throughout this paper. In addition we use a different numerical method, the Wiener bundle method, to compute values of the scalar field. This serves as a check of our random walk methods, and also sets the stage for our application of the method of shadowing from dynamical systems theory. We introduce a fictitious 'zerodiffusivity' solution as a heuristic tool in demonstrating the role of the underlying geometrical structures in the flow and in interpreting the role of slow mixing as a local smoothing of fine-scale structure in the scalar field, created by the stirring process.

The hyperbolic invariant set (Smale 1967) associated with Smale horseshoes (Smale 1967 ) is the prototype of a chaotic dynamical system, and the shadowing lemma (Bowen 1975) from dynamical systems theory is one of the fundamental results for the dynamics on a hyperbolic invariant set. Recent work of Klapper (1992a) has used shadowing theory (Anosov 1967; Bowen 1975) to study the small-diffusivity scalar advection-diffusion problem. Asymptotic results were obtained for the restricted class of uniformly hyperbolic systems, and therefore apply to typical chaotic processes in only a non-rigorous sense. Justification (Klapper $1992 a$ ) for its validity is based on existing numerical evidence (Hammel, Yorke \& Grebogi 1987, 1988; Grebogi et al. 1990) that typical chaotic dynamical processes have the shadowing property. In a rough sense, a dynamical system that has the shadowing property is guaranteed to have a deterministic orbit that remains close to any noisy orbit with bounded noise, where how 'close' depends on the noise level. The shadowing property has been used previously to reduce bounded additive noise in orbits generated by chaotic dynamical 
systems (Hammel 1990; Farmer \& Sidorowich 1991). That the shadowing property can be used to treat scalar diffusion is not surprising since diffusion can be regarded as a noisy component in the kinematics of a diffusive tracer.

We use these ideas to develop a qualitative understanding of our random-walk solutions of the time-dependent scalar field and the interplay between the stirring and mixing processes. It is found that increased chaotic advection produces more localized and non-uniform distributions, even in regions of the flow that have no islands of stability bounded by invariant closed curves; near integrability, such curves will be provided by Kolmogorov-Arnold-Moser (KAM) tori and island bands, but as one perturbs the dynamical system further away from integrability there are no surviving invariant closed curves, and we choose such a parameter-regime to emphasize our result. The phenomenon contrasts sharply with that in the case of chaotic advection in bounded domains where the chaotic particle motion promotes rapid homogenization (Jones 1991) of diffusive tracer, giving rise to an asymptotically uniform scalar distribution. Reasons for this phenomenon are sought using shadowing theory. The method of shadowing also helps us understand that the form of the asymptotic distribution and the time-scale over which it is attained is also intimately linked with the geometrical structures in the flow, and the connection is made explicit by considering the details of exact dynamical trajectories that 'shadow' the 'noisy' Wiener trajectories of a diffusive tracer. We also show how the presence of the plane wall and the consequent regular dynamics of particles in a narrow near-wall region strongly influences the time-evolution of the scalar field, thus underlining the role of non-hyperbolicity at the wall. In engineering applications the flux of a passive scalar, integrated over the active surface, is a quantity of considerable practical importance; our computations show it is largely independent of the details of the chaotic advectiondiffusion phenomena above the wall, for the class of flows considered. Perturbation arguments in the thermal boundary layer adjacent to the active no-slip surface further clarify this issue.

The organization of this paper is as follows. In $\S 2$ we construct an approximate representation of a chaotic separation bubble over a plane wall. In $\S 3$ we set up the scalar advection-diffusion problem. Numerical schemes are developed to obtain finite-time solutions of the large-Péclet-number or small-diffusivity advection-diffusion problem. In $\S 4$, a 'zero-diffusivity' solution is constructed by solving for the scalar field in the thermal boundary layer at the wall at small time and treating this as an 'initial distribution' of perfect tracers which is subsequently stirred but not mixed by chaotic advection. In $\S 5$ we apply shadowing theory to our scalar advection-diffusion problem. We end with a discussion and concluding remarks in $\S 6$.

\section{Time-periodic separation bubble at a plane wall}

We first construct a viscous, incompressible, two-dimensional flow that has the topology of a steady separation bubble at a plane wall in the form of a Taylor-series expansion from a point on the no-slip boundary. The construction suggests ways in which time-dependent terms can be introduced in the vector field such that one obtains an asymptotically exact representation of a time-periodic bubble with chaotic particle trajectories. The advantage of the Taylor-series expansion method (Perry \& Chong $1986 a$ ) is that one can generate boundary layer flows, especially separation patterns with desired topological features, as local Taylor-series expansions to arbitrary order, without regard to the outer inviscid flow. The method assumes the solutions of the continuity and Navier-Stokes equations for incompressible flow are smooth. 
A point on the plane wall is chosen to be the origin for two-dimensional rectangular coordinates $\left(x_{1}, x_{2}\right)$, where $x_{1}$ is a coordinate along the wall and $x_{2}$ is the coordinate normal to the wall. The velocity vector $\boldsymbol{u}(x, t), \boldsymbol{x} \equiv\left(x_{1}, x_{2}\right) \in \mathbb{R}^{1} \times \mathbb{R}^{+}, \boldsymbol{u} \in \mathbb{R}^{2}$, is written in the form of an asymptotic third-order expansion from the origin

$$
u_{i}=A_{i}+A_{i j} x_{j}+A_{i j k} x_{j} x_{k}+A_{i j k l} x_{j} x_{k} x_{l}+O(4),
$$

where $O(m)$ represents a homogeneous polynomial of degree $n \geqslant m$. The $i, j, k, \ldots$ can take values of 1 or 2 , since the flow is two-dimensional. The coefficients $A_{i}, A_{i j}$, etc. are functions of time if the flow is unsteady, and they are symmetric tensors in all indices except the first. The number of independent coefficients, $N_{c}$, in our two-dimensional third-order expansion is easily determined (Perry \& Chong 1986 $a$ ), $N_{c}=20$. The basic idea behind the method is first to force the tensor coefficients $A_{i j k \ldots}$ to satisfy the continuity equation and the boundary conditions. This result for our application is that six tensor coefficients remain. Imposing the Navier-Stokes equation yields one additional constraint, the following ordinary differential equation (see Perry \& Chong $1986 b)$ :

$$
\dot{A}_{12}=6 v\left[2 A_{1112}+A_{1222}\right],
$$

where $v$ is the kinematic viscosity of the fluid. The remaining degrees of freedom are determined using the topological constraints that are prescribed by the desired topology of the separation pattern.

Following Perry \& Chong $(1986 a)$, we specify the boundary vorticity $w\left(x_{2}=0\right)$ to vary according to the equation

$$
w\left(x_{2}=0\right) \equiv-\left.\frac{\partial u_{1}}{\partial x_{2}}\right|_{x_{2}=0}=-K\left(x_{1}^{2}-x_{s}^{2}\right),
$$

with $K>0$, thus generating two points of zero shear stress on the wall at $\left(-x_{s}, 0\right)$ and $\left(+x_{s}, 0\right)$, which act as a point of separation and a point of attachment, respectively. We note that in the time-dependent flow the parameter $K$ can be a function of time. At this point the asymptotic expansion of (2.1) reduces to

$$
\left.\begin{array}{l}
u_{1}=-K x_{s}^{2} x_{2}+A_{122} x_{2}^{2}+K x_{1}^{2} x_{2}+3 A_{1122} x_{1} x_{2}^{2}+A_{1222} x_{2}^{3}+O(4), \\
u_{2}=-K x_{1} x_{2}^{2}-A_{1122} x_{2}^{3}+O(4) .
\end{array}\right\}
$$

Consider first the time-independent problem. At steady state, (2.2) and (2.3) yield

$$
A_{1222}=-2 K / 3 \text {. }
$$

There are now only two independent unknown coefficients. These are specified using the two topological constraints that (2.4) must satisfy in order that the steady flow field has the topology of a symmetrical (about the origin) separation bubble, and these are: (i) symmetry condition on $u_{2}$, requiring $u_{2}\left(x_{1}=0, x_{2}\right)=0$, (ii) elliptic stagnation point at $\left(0, x_{2}^{*}\right), 0<x_{2}^{*} \ll 1$. Condition (i) specifies $A_{1122}=0$. Substituting these in (2.4) and applying condition (ii) gives

$$
\begin{aligned}
& -K x_{s}^{2}+A_{122} x_{2}^{*}-\frac{2 K}{3} x_{2}^{*^{2}}=0, \\
& -K x_{s}^{2}+2 A_{122} x_{2}^{*}-2 K x_{2}^{*^{2}}>0,
\end{aligned}
$$

which yields an elliptic stagnation point on the $x_{2}$-axis, located at $x_{2}^{*} \in\left(0,(3 / 2)^{1 / 2} x_{s}\right)$ for $A_{122} \in\left((8 / 3)^{1 / 2} K x_{s}, \infty\right)$. As $A_{122} \rightarrow \infty$, the elliptic stagnation point approaches the wall, i.e. $x_{2}^{*} \rightarrow 0$, and the bubble shrinks closer to the wall. As $A_{122} \rightarrow(8 / 3)^{1 / 2} K x_{s}$ 
from above, $x_{2}^{*} \rightarrow(3 / 2)^{1 / 2} x_{s}$ from below and the bubble grows in size. For $A_{122}<$ $(8 / 3)^{1 / 2} K x_{s}$, there are no stagnation points in the entire domain of the flow. We note that for $A_{122} \in\left((8 / 3)^{1 / 2} K x_{s}, \infty\right)$ there is also a hyperbolic stagnation point or saddle located at $\left(0, \bar{x}_{2}\right)$, and $\bar{x}_{2} \in\left((3 / 2)^{1 / 2} x_{s}, \infty\right)$; as $A_{122} \rightarrow \infty, \bar{x}_{2} \rightarrow \infty$ and the separation between the two stagnation points is maximum, while as $A_{122} \rightarrow(8 / 3)^{1 / 2} K x_{s}$ from above, $\bar{x}_{2} \rightarrow(3 / 2)^{1 / 2} x_{s}$ from above so that at $A_{122}=(8 / 3)^{1 / 2} K x_{s}$ the hyperbolic and elliptic stagnation points coalesce and disappear as $A_{122}$ decreases below $(8 / 3)^{1 / 2} K x_{s}$. At $A_{122}=3 K x_{s}$, the elliptic stagnation point is located at $(0,0.3625)$. The hyperbolic stagnation point or saddle is located at $(0,4.1374)$. It is therefore sufficiently far away from the wall to have no bearing on passive scalar transport close to the wall and is ignored hereafter. With this choice of $A_{122}$, the time-independent velocity field is completely specified,

$$
\left.\begin{array}{l}
u_{1}=-K x_{s}^{2} x_{2}+3 K x_{s} x_{2}^{2}+K x_{1}^{2} x_{2}-\frac{2}{3} K x_{2}^{3}+O(4), \\
u_{2}=-K x_{1} x_{2}^{2}+O(4),
\end{array}\right\}
$$

and is a low-order approximation of a steady two-dimensional separation bubble at a plane wall. By varying the coefficient $A_{122}$, the size of the steady separation bubble can be varied with important consequences on the associated advection-diffusion problem, and is the subject of $\S 3.6$. At present we continue with our choice of $A_{122}=3 K x_{s}$. The streamlines (or pathlines) corresponding to the steady velocity field of (2.5) are shown in figure 1. From a dynamical systems viewpoint, the phase space of (2.5) has a heteroclinic connection $\psi_{h}$ between the point of separation and the point of attachment, separating bounded and unbounded motion, where $\psi_{h}$ is the value of the time-independent stream function (obtained below) on the separatrix. Introducing a time-periodic perturbation in (2.5) is expected to destroy this degenerate structure, giving rise to chaotic particle motion. An obvious way is to break the symmetry in the steady state. To do so we let $A_{1122}(t)=K \beta \sin (\omega t)$. The remaining coefficients are left unchanged. From (2.2), $K$ remains independent of $t$ and hence all remaining tensor coefficients are also time-independent. Non-dimensionalizing, $u_{i} \rightarrow u_{i} K x_{s}^{3}, x_{i} \rightarrow x_{i} x_{s}$, $t \rightarrow t / K x_{s}^{2}, \omega \rightarrow \omega K x_{s}^{2}$, the time-dependent velocity field becomes

$$
\left.\begin{array}{l}
u_{1}=-x_{2}+3 x_{2}^{2}+x_{1}^{2} x_{2}-\frac{2}{3} x_{2}^{3}+3 \beta x_{1} x_{2}^{2} \sin (\omega t)+O(4), \\
u_{2}=-x_{1} x_{2}^{2}-\beta x_{2}^{3} \sin (\omega t)+O(4) .
\end{array}\right\}
$$

The stream function $\psi$ is easily obtained,

$$
\psi\left(x_{1}, x_{2}, t\right)=-\frac{x_{2}^{2}}{2}+x_{2}^{3}+\frac{x_{1}^{2} x_{2}^{2}}{2}-\frac{x_{2}^{4}}{6}+\beta x_{1} x_{2}^{3} \sin (\omega t)+O(5) .
$$

Truncated to third order, (2.6) can be expressed in the form

$$
u(x, t)=f^{u}(x)+\beta g^{u}(x) \sin (\omega t),
$$

where $f^{u} \equiv\left(f_{1}^{u}, f_{2}^{u}\right)=\left(-x_{2}+3 x_{2}^{2}+x_{1}^{2} x_{2}-\frac{2}{3} x_{2}^{3},-x_{1} x_{2}^{2}\right), g^{u} \equiv\left(g_{1}^{u}, g_{2}^{u}\right)=\left(3 x_{1} x_{2}^{2},-x_{2}^{3}\right)$, and $\beta$ can be considered as the perturbation amplitude, while $\omega$ is the frequency of the perturbation. The associated Poincare map or time- $T$ map, $T=2 \pi / \omega$, which is defined in the usual way in $\S 3$, shows highly irregular motion in the bubble region indicating chaotic particle trajectories. In physical terms, the time-periodic perturbation in (2.8) that leads to bubble break-up and chaotic trajectories in the viscous boundary layer can be attributed to an oscillatory outer inviscid flow. In particular, it should be noted that the chaotic advection field of (2.8) does not arise from any inherent oscillatory instability in the equations of motion at large Reynolds 


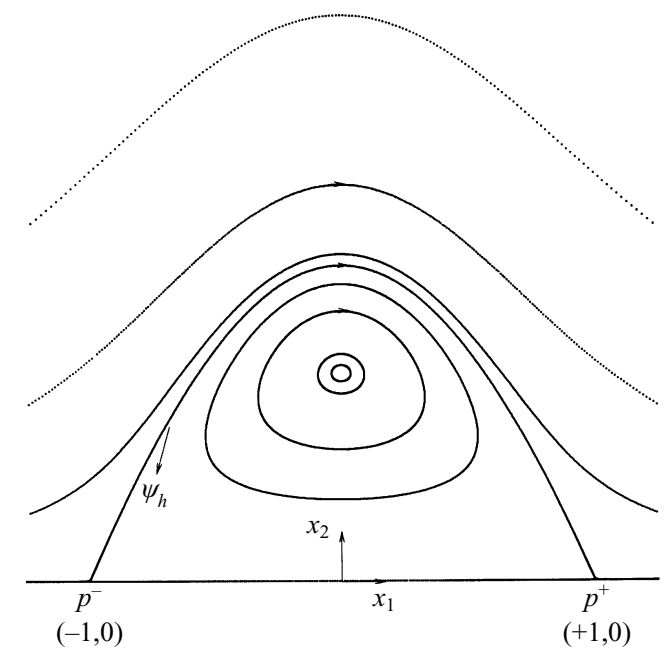

FIGURE 1. Streamlines for the steady two-dimensional separation bubble at a plane wall, obtained using (2.5).

number but, instead, arises from an external forcing presumably caused by the outer inviscid flow. The Reynolds number, $R e=K x_{s}^{4} / v$, for our boundary layer flow is arbitrary, though it must be noted that the region of accuracy of the truncated series solution shrinks as the Reynolds number is increased (Perry \& Chong 1986a).

Finally, there are alternative schemes for introducing a time-periodic perturbation in (2.5) such that the time-dependent velocity field remains an asymptotically exact solution of the Navier-Stokes and continuity equations, and again gives rise to chaotic particle trajectories; there is of course no unique representation of a two-dimensional chaotic advection field adjacent to a no-slip boundary. However we shall show that the qualitative aspects of the associated passive scalar advection-diffusion problem depend primarily on certain generic structures in these chaotic advection fields, regardless of the specific form of the equations giving rise to the chaotic particle motion. Section 3.5 deals with this issue by considering an alternative local representation of a chaotic advection field.

\section{Advection-diffusion of a passive scalar at small diffusivity or large Péclet number}

A localized portion of the plane no-slip boundary is considered as an active surface. In the case of heat transfer, the active surface can be considered as a local 'hot spot' at the wall and is modelled as a local step change in temperature with the step placed symmetrically about the unperturbed bubble. At time $t=0$ the temperature has a dimensionless value of unity at the wall over the dimensionless interval $x_{1} \in[-1.5,1.5]$ and is zero everywhere else on the wall and in the fluid. The temperature distribution at the wall is maintained externally and serves as a time-independent boundary condition as heat diffuses from the wall and into the fluid. Non-dimensionalizing time $t$ using the advection time-scale $1 / K x_{s}^{2}$ gives the familiar evolution equation for the advection-diffusion of a scalar field $\theta(x, t)$, which can be considered as temperature,

$$
\frac{\partial \theta}{\partial t}+\boldsymbol{u} \cdot \nabla \theta=\frac{1}{P e} \nabla^{2} \theta,
$$


where the Péclet number $P e=K x_{s}^{4} / D$, with $D$ the scalar diffusivity. The advecting two-dimensional velocity field $u(x, t)$ is given by $(2.8)$. The following initial and boundary conditions:

$$
\begin{aligned}
\theta\left(x_{1}, x_{2}>0, t=0\right) & =0, \\
\theta\left(x_{1}, x_{2}=0, t\right) & =1-H\left(\left|x_{1}\right|-1.5\right), \\
\theta\left(\left|x_{1}\right| \rightarrow \infty, x_{2}>0, t\right) & =0, \\
\theta\left(x_{1}, x_{2} \rightarrow \infty, t\right) & =0,
\end{aligned}
$$

with $\left(x_{1}, x_{2}\right) \in \mathbb{R}^{1} \times \mathbb{R}^{1}$, completely specify the advection-diffusion problem. $H(\cdot)$ is the Heaviside step function. In the absence of scalar diffusion the deterministic Lagrangian motion of a passive scalar is described by the velocity field. Two-dimensional timeperiodic flows have been studied extensively using a dynamical systems approach (e.g. see Wiggins 1992) and the geometrical structures that arise naturally in the domain of the flow, such as invariant manifolds, homoclinic/heteroclinic tangles, hyperbolic invariant sets and KAM tori are well known. However, it is still far from clear how these structures influence the transport and distribution of a passive scalar in the presence of scalar diffusion. Our model problem provides a convenient framework for understanding some of the transport issues that arise naturally in such flows. The domain of interest is the flow region immediately above the active portion of the wall and since we will be making frequent reference to this region, we loosely term it as the 'bubble-region'.

In the context of dynamical systems theory, the velocity field of (2.8) is a timeperiodic perturbation of a planar Hamiltonian vector field, where the stream function of (2.7) plays the role of the Hamiltonian. The analysis of the global structure of the flow is most clearly carried out by studying the associated Poincare map, which is the time- $T$ map obtained by considering the discrete motion of points in timeintervals of one period $T$ of the perturbation, and since the perturbation is also Hamiltonian the Poincare map is area-preserving. In this case one would expect Smale horseshoes, resonance bands and KAM tori to arise in the phase space of (2.8), which is also the physical space of the flow. However, from the dynamical systems viewpoint, our problem is non-generic because every point on the no-slip boundary is a non-hyperbolic stagnation point, despite the fact that it is a commonly encountered situation in fluid flows. Consequently, the mathematical theorems (see Wiggins 1990, 1992) proving existence of chaotic dynamics do not apply. For the case of hyperbolic stagnation points the existence of stable and unstable manifolds is familiar, but the non-hyperbolic case requires special consideration. In the unperturbed $(\beta=0)$ integrable system, the bubble has a point of separation, denoted by $p^{-}$, a point of attachment, denoted by $p^{+}$, and a heteroclinic connection $\psi_{h}$ between $p^{-}$and $p^{+}$. The points $p^{+} \equiv(+1,0)$ and $p^{-} \equiv(-1,0)$, which are the points of zero shear stress on the wall, are non-hyperbolic stagnation points. The standard scheme of introducing the phase of the periodic perturbation in (2.8) gives an autonomous vector field

$$
\begin{aligned}
\dot{x} & =f^{u}(x)+\beta g^{u}(x) \sin (\phi), \\
\dot{\phi} & =\omega,
\end{aligned}
$$

where the phase space of the autonomous system is now $\mathbb{R}^{2} \times S^{1}$. For $\beta=0$, when viewed in the three-dimensional phase space $\mathbb{R}^{2} \times S^{1}, p^{+}$and $p^{-}$become periodic 
orbits

$$
\gamma^{ \pm}(t)=\left(p^{ \pm}, \phi(t)=\omega t\right)
$$

with a two-dimensional stable and two-dimensional unstable manifold respectively, denoted by $W^{s}\left(\gamma^{+}(t)\right)$ and $W^{u}\left(\gamma^{-}(t)\right)$. Therefore, $W^{s}\left(\gamma^{+}(t)\right)$ and $W^{u}\left(\gamma^{-}(t)\right)$ coincide along a two-dimensional heteroclinic manifold, $\Gamma_{\gamma}$. For $\beta \neq 0$, it is easily verified from (2.8) that $p^{ \pm}$persist as the points of zero shear stress on the wall, which implies $p_{\beta}^{ \pm}=p^{ \pm}$(Ghosh 1994; Shariff, Pulliam \& Ottino 1991), and hence the periodic orbits for $\beta \neq 0$ are $\gamma_{\beta}^{ \pm}(t)=\gamma^{ \pm}(t)$. However, we note that the invariant manifold theorem (see Theorem 4.1, Hirsch, Pugh \& Shub 1977) for the persistence of normally hyperbolic invariant manifolds and the persistence and smoothness of their stable and unstable manifolds (at sufficiently small $\beta$ ) does not apply to $\gamma^{ \pm}(t)$; in our problem, persistence of the invariant manifolds is decided by computation of the corresponding invariant manifolds of the associated Poincare map, which we shall define momentarily. Assuming $W^{s}\left(\gamma^{+}(t)\right)$ and $W^{u}\left(\gamma^{-}(t)\right)$ persist for $\beta \neq 0$, the stable and unstable manifolds of $\gamma_{\beta}^{+}(t)$ and $\gamma_{\beta}^{-}(t)$, denoted by $W^{s}\left(\gamma_{\beta}^{+}(t)\right)$ and $W^{u}\left(\gamma_{\beta}^{-}(t)\right)$ respectively, will generically not coincide. The Poincaré map is defined as a global cross-section in the usual way,

$$
\begin{aligned}
P_{\beta}^{\bar{\phi}}: \Sigma^{\bar{\phi}} & \rightarrow \Sigma^{\bar{\phi}}, \\
\left(x_{1}(\bar{\phi}), x_{2}(\bar{\phi})\right) & \mapsto\left(x_{1}(\bar{\phi}+2 \pi), x_{2}(\bar{\phi}+2 \pi)\right) .
\end{aligned}
$$

The intersection with $\Sigma^{\bar{\phi}}$ of the stable and unstable manifolds of $\gamma_{\beta}^{+}(t)$ and $\gamma_{\beta}^{-}(t)$ respectively are denoted as

$$
\begin{aligned}
& W_{\beta}^{s}(\bar{\phi}) \equiv W^{s}\left(\gamma_{\beta}^{+}(t)\right) \cap \Sigma^{\bar{\phi}}, \\
& W_{\beta}^{u}(\bar{\phi}) \equiv W^{u}\left(\gamma_{\beta}^{-}(t)\right) \cap \Sigma^{\bar{\phi}} .
\end{aligned}
$$

Rom-Kedar, Leonard \& Wiggins (1990) considered the transport of a passive scalar in the absence of scalar diffusion in a similar problem and found the unstable manifold, i.e. $W_{\beta}^{u}(\bar{\phi})$, as the dominant organizing structure. They considered the evolution of an arbitrary blob of fluid under several iterations of the Poincare map and showed that the unstable manifold behaves in some sense as an attractor since neighbourhoods of $W_{\beta}^{u}(\bar{\phi})$ are stretched along $W_{\beta}^{u}(\bar{\phi})$ and flattened in a complementary direction under forward iterations of $P_{\beta}^{\bar{\phi}}$. Of course, because of the area-preserving property of $P_{\beta}^{\bar{\phi}}$ that derives from the incompressibility of the fluid, $W_{\beta}^{u}(\bar{\phi})$ is not an attractor in the usual sense. Our main goal in this section is to motivate the following: for scalar transport with small scalar diffusivity, the unstable manifold $W_{\beta}^{u}(\bar{\phi})$ continues to be the organizing structure in the distribution of the scalar field and the time evolution of the scalar field for the $\beta \neq 0$ case can be understood in terms of the structure of $W_{\beta}^{u}(\bar{\phi})$. Additionally, there are other important considerations, some of which have been outlined in the Introduction: for example the role of hyperbolicity and the strong stretching and folding properties characteristic of chaotic advection fields, the role of non-hyperbolicity at the wall, the asymptotic state of the scalar distribution, etc., and to what extent they contribute to our understanding of the advection-diffusion process and its interplay with the geometrical structures in the flow. As a first step towards investigating these issues, finite-time distributions of the scalar field are obtained by solving (3.1) numerically. We confine our attention entirely to the case of small scalar diffusivity, or large Péclet number; large scalar diffusivity would result 
in a diffusively dominant advection-diffusion process, in which case the role of the geometrical structures in the flow can be expected to be less prominent.

\subsection{A random-walk scheme for solving the advection-diffusion equation}

The singular limit of large Péclet number is not easily tackled by any numerical scheme that embraces the Eulerian approach and tries to solve the partial differential equation of (3.1) directly. At large Pe, a thermal boundary layer will form in the vicinity of the active surface of thickness proportional to $P e^{-1 / 3}$ (see $\S 3.4$ ), which places severe constraints on the spatial resolution obtainable in the boundary layer in a finite-difference solution of (3.1) at large Pe. In contrast, a random-walk solution uses a Lagrangian approach based on the stochastic differential corresponding to the scalar advection-diffusion equation. Saffman (1959) used a random-walk approach to study the effects of molecular diffusion on the transport of a passive scalar in flow through porous media. Chorin (1973) extended the random-walk approach to numerical solutions of the vorticity diffusion equation corresponding to the NavierStokes equations for incompressible flow in two space dimensions at large Reynolds numbers, where the problem is complicated by the fact that the vorticity is not a passive scalar. Since we require solutions to (3.1) at Péclet numbers so large that finite-difference methods are difficult to apply, a random-walk or Brownian motion scheme is developed below for solving the scalar advection-diffusion problem.

The active boundary is imagined to be a constant temperature bath. The physical process of diffusion of passive scalar from the active portion of the plane boundary into the flow is imitated by numerically simulating a constant temperature bath. The bath has width identical to the extent of the active surface and depth chosen according to the Péclet number. At $t=0, N$ points are randomly distributed in the bath at locations $r_{i}^{0}=\left(x_{1 i}^{0}, x_{2 i}^{0}\right), i=1, \ldots, N, N$ large, each of mass $1 / N_{d}$ where $N_{d}=N / A_{\text {bath }}$ is the number density of the randomly distributed points, and $A_{b a t h}$ is the area of the constant temperature bath. The evolution of temperature in the bath is governed by the simple transient diffusion equation

$$
\frac{\partial \theta}{\partial t}=\frac{1}{P e} \nabla^{2} \theta
$$

with initial data $\theta(x, t=0)=1$ and adiabatic boundary conditions, which of course has solution $\theta(x, t)=1$. The probabilistic interpretation of the transient diffusion equation with given initial data is well known (Chandrasekhar 1943). For a brief survey with view to applications in numerical schemes based on random-walk methods, see Chorin \& Marsden (1979). The stochastic differential corresponding to (3.2) is simply

$$
\mathrm{d} x=(2 / P e)^{1 / 2} \mathrm{~d} W_{t},
$$

where $W_{t}$ is a two-dimensional Wiener process (Arnold 1974). Hence, in every timestep $\Delta t$, the points may be advanced according to

$$
x_{1 i}^{n+1}=x_{1 i}^{n}+\eta, x_{2 i}^{n+1}=x_{2 i}^{n}+\xi, n=0,1, \ldots,
$$

where $\eta, \xi$ are Gaussianly distributed random variables, each with mean zero and variance $2 \Delta t / P e$, and $x_{1 i}^{n} \equiv x_{1 i}(n \Delta t), x_{2 i}^{n} \equiv x_{2 i}(n \Delta t)$. Except for the boundary that coincides with the wall, the remaining three boundaries of the constant temperature bath behave as adiabatic surfaces and are therefore treated as perfectly reflecting boundaries (Chandrasekhar 1943). As for the wall, when a point jumps across the wall and into the domain of the flow, a new point of identical mass is created at the reflection point inside the bath. To ensure that the reflection point is almost always 
- i.e. with probability arbitrarily close to unity - inside the bath, it is sufficient to choose the depth of the bath as $10 \sigma$ where $\sigma$ is the standard deviation of the Gaussian 'jumps'. In every time-step, a constant number density $N_{d}$ of randomly distributed points of masses $1 / N_{d}$ is maintained inside the bath and it therefore simulates a constant temperature bath. Points that jump across the wall, from the bath and into the domain of the flow, have trajectories generated by the stochastic differential corresponding to (3.1)

$$
\mathrm{d} x=u \mathrm{~d} t+(2 / P e)^{1 / 2} \mathrm{~d} W_{t} .
$$

A point in the domain of the flow, i.e. at location $r^{0}=\left(x_{1}^{0}, x_{2}^{0}\right)$ with $x_{2}^{0}>0$, is therefore advanced according to

$$
x_{1}^{n+1}=x_{1}^{n}+\Delta x_{1}^{n}+\eta, x_{2}^{n+1}=x_{2}^{n}+\Delta x_{2}^{n}+\xi,
$$

where

$$
x_{1}^{n}=x_{1}(n \Delta t), x_{2}^{n}=x_{2}(n \Delta t), \Delta x_{1}^{n}=\int_{n \Delta t}^{(n+1) \Delta t} u_{1} \mathrm{~d} t, \Delta x_{2}^{n}=\int_{n \Delta t}^{(n+1) \Delta t} u_{2} \mathrm{~d} t,
$$

and $\eta, \xi$ are Gaussianly distributed random variables as before; the velocity field is integrated using fourth-order Runge-Kutta scheme. The Gaussianly distributed random variables $\eta$ and $\xi$ are obtained from uniformly distributed computer-generated pseudo-random numbers using standard algorithms based on the Box-Mueller transformation (e.g. see Press et al. 1986). The increment $\left(\Delta x_{1}^{n}, \Delta x_{2}^{n}\right)$ is the deterministic jump in a time-step $\Delta t$ arising from the continuum velocity of a fluid particle, while $(\eta, \xi)$ is a random component describing the Brownian motion of the molecules. For the advection-diffusion process in the domain of the flow, the wall specifies a constant temperature boundary condition and can therefore be treated as a perfectly absorbing boundary (Chandrasekhar 1943). Hence, trajectories generated by (3.3) that cross the wall, which is signalled by $x_{2}^{n} \leqslant 0$, are terminated. After $n$ steps, the expected distribution of mass on the upper half-plane, $x_{2}>0$, gives the temperature field at time $t=n \Delta t$. Therefore the temperature at time $t$ at a point $\left(x_{1}, x_{2}\right), x_{2}>0$, is given by

$$
\theta\left(x_{1}, x_{2}, t\right)=\lim _{N \rightarrow \infty} \frac{\text { number of points contained in } I_{1}\left(x_{1}\right) \times I_{2}\left(x_{2}\right) \text { at time } t}{N_{d} A_{b o x}},
$$

where $I_{1}\left(x_{1}\right)=\left[x_{1}-\frac{1}{2} \Delta x_{1}, x_{1}+\frac{1}{2} \Delta x_{1}\right], I_{2}\left(x_{2}\right)=\left[x_{2}-\frac{1}{2} \Delta x_{2}, x_{2}+\frac{1}{2} \Delta x_{2}\right], I_{1}\left(x_{1}\right) \times I_{2}\left(x_{2}\right)$ is the Cartesian product of the two closed intervals, and $A_{b o x}=\Delta x_{1} \Delta x_{2}$. The flux across the wall, integrated over the active portion of the wall, is given by the expected rate at which points cross the active portion of the wall (Chandrasekhar 1943). The wall-integrated flux $J_{w}$ at time $t=n \Delta t$ is simply

$$
J_{w}(t)=\lim _{N \rightarrow \infty} \frac{\Delta N(n)}{N_{d} \Delta t},
$$

where $\Delta N(n)$ is the net number of points crossing the active portion of the wall at the $n$th time-step. Therefore $\Delta N(n)$ is the number of points that jump from the bath and into the flow at the $n$th time-step minus the number of points in the domain of the flow that jump across the active surface at the $n$th time-step and are terminated. We compute the wall-integrated flux averaged over time $T=2 \mathrm{k} \Delta t$, which is given by

$$
\left\langle J_{w}(t)\right\rangle_{T}=\lim _{N \rightarrow \infty} \frac{\Delta N(n, k)}{N_{d}(2 k \Delta t)},
$$

where $\Delta N(n, k)$ is the net number of points crossing the active surface in $2 k$ time-steps, 
from time $(n-k) \Delta t$ to $(n+k) \Delta t$. Therefore $\left\langle J_{w}(t)\right\rangle_{T}$ is in fact

$$
\left\langle J_{w}(t)\right\rangle_{T}=\frac{1}{T} \int_{t-T / 2}^{t+T / 2}\left(\int_{x_{1}=-1.5}^{x_{1}=1.5}-\left.\frac{1}{P e} \frac{\partial \theta}{\partial x_{2}}\left(x_{1}, x_{2}, s\right)\right|_{x_{2}=0} \mathrm{~d} x_{1}\right) \mathrm{d} s .
$$

Standard error-estimation techniques (Ghosh 1994) show the error in the computed scalar field $\theta\left(x_{1}, x_{2}, t\right)$ is $O\left(\left(N_{d} A_{b o x}\right)^{-1 / 2}\right)$. The consequent slow convergence, which is typical of random-walk methods, requires that $N$ be very large especially when high spatial resolution, i.e. small $\Delta x_{1} \Delta x_{2}$ or small $A_{b o x}$, is desired. In our computations $N$ is $O\left(10^{6}\right)$. However, it is not hard to realize that the random-walk scheme described above is ideally suited for parallel computation and an implementation of the method on a parallel computer like the 512 node Intel Touchstone Delta reduces computation time by several orders of magnitude. In fact, our code can use any number of compute nodes and proceeds in parallel by distributing $N$ equally among the number of available nodes, with an absolute minimum of node interactions. We note that there are alternative random-walk schemes that can be formulated for our advectiondiffusion problem, but these were discarded in favour of the scheme described above on account of the ease with which the computations can be rendered parallel.

\subsection{The Wiener bundle method}

The results obtained using the random-walk method above were verified by comparing the solution of the scalar field at several points in the domain of interest against point values computed using an independent numerical scheme also based on the theory of Brownian motion, called the Wiener bundle method. The method has been used previously to study heat conduction problems (Haji-Sheikh \& Sparrow 1967), to treat the advection-diffusion of magnetic field in magnetohydrodynamics (Molchanov, Ruzmaikin \& Sokolov 1985; Klapper $1992 b$ ), and in the study of chaotic advection of scalars (Klapper 1992a). While the Wiener bundle method is generally inefficient for whole field computations, it has the unique advantage of allowing calculation of the scalar value at a single point without calculating the whole field. Therefore, when only a few point values are desired the method offers a distinct advantage over other numerical schemes. The Wiener bundle method will also be used in $\S 5$ to gain useful insights into the small diffusivity scalar advection-diffusion problem, and we therefore outline it briefly. The method is also called a pseudo-Lagrangian method since it is a generalization of the Lagrangian solution for zero diffusivity. At $P e=\infty$, i.e. for the case of zero-diffusivity, (3.1) is solved by considering $\theta$ as constant along pathlines in the flow. Hence $\theta\left(x\left(x^{0}, t\right), t\right)=\theta\left(x^{0}, t_{0}\right), t>t_{0}$, where $x\left(x^{0}, t\right)$ is the Lagrangian variable defined by $\mathrm{d} x=u \mathrm{~d} t, x\left(x^{0}, t_{0}\right)=x^{0}$. The zero-diffusivity Lagrangian solution can be generalized to the case of non-zero diffusivity, i.e. finite $P e$, by averaging over a bundle of random (Wiener) trajectories. A Wiener trajectory is generated using the stochastic differential of (3.3). Therefore, starting at a point $x$ at time $t$ and integrating backwards in time using (3.3) yields a 'bundle' of Wiener trajectories, each of which starts from a random point $x^{0}$ at initial time $t_{0}$ and reaches $x$ at time $t$. Each Wiener trajectory 'carries' with it the scalar value $\theta$ of the point from which it originates, just as in the zero-diffusivity Lagrangian solution, and an expectation taken over the bundle of Wiener trajectories determines $\theta(x, t)$. The Wiener bundle solution of (3.1) is given by

$$
\theta(x, t)=\left\langle\theta\left(x^{0}, t_{0}\right)\right\rangle
$$



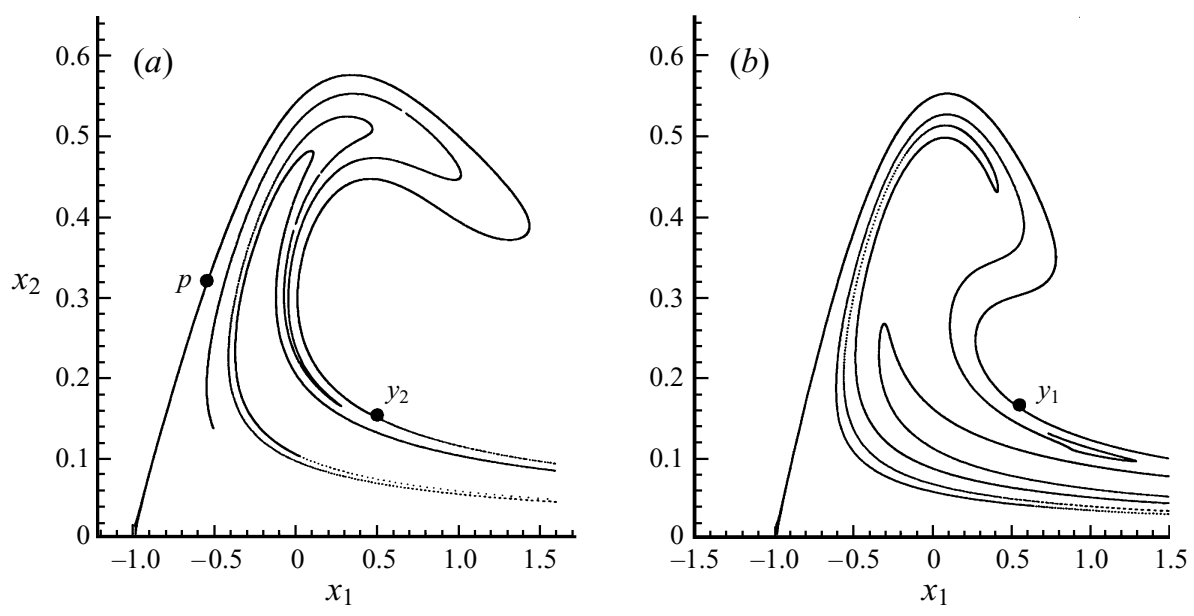

FIgURE 2. The unstable manifold $W_{\beta}^{u}$ at $\omega=0.72$ and $(a) \beta=0.6,(b) \beta=0.2$.

where the average is taken over all Wiener trajectories starting at a random point $x^{0}$ and ending at a given non-random point $x$ in time $t-t_{0}$. It is easy to verify that (3.5) is indeed a solution of (3.1). For a rigorous proof, see McKean (1969).

\subsection{Scalar distributions - numerical simulation results}

The unstable manifold of $p^{-}$for the Poincare map corresponding to the cross-section $\bar{\phi}=0, P_{\beta} \equiv P_{\beta}^{\bar{\phi}=0}$, denoted $W_{\beta}^{u} \equiv W_{\beta}^{u}(\bar{\phi}=0)$, is computed for two different values of the perturbation amplitude, $\beta=0.2,0.6$, keeping the frequency of the perturbation fixed at $\omega=0.72$, and these are shown in figure 2. Finite-time distributions of the scalar field at $P e=2.5 \times 10^{4}$ are obtained numerically on the 512 node Intel Delta using the random-walk method for whole field computations described earlier. The scalar field is computed at several times that are integral multiples of the perturbation period at again $\beta=0.2,0.6$, and these are displayed in figures $3(a-d), 4(a-d)$. The time-evolution of the scalar field is also obtained for $\beta=0$, i.e. for the steady bubble, at the same $P e$ for the sake of comparison and is shown in figure 5(a-d). For the steady bubble the asymptotic or long-time scalar field distribution is expected to be uniform in the circulation or core region of the bubble (Batchelor 1956; Pan \& Acrivos 1968), while there will be boundary layers along the wall and straddling the separatrix, $\psi_{h}$. The diffusive time-scale, being of the order of the Péclet number, is very large and since the asymptotic distribution is attained over the diffusion timescale (Rhines \& Young 1983) it would take a prohibitively long-time computation to reproduce the expected asymptotic distribution. However, it is clear from figure $5(a-d)$ that the distribution of the scalar field for the case of steady $(\beta=0)$ advection is tending to the expected asymptotic distribution as $t$ increases. Comparing figures 3 , 4 with figure 5 , it is clearly evident that the evolution of the scalar field for the $\beta \neq 0$ case is markedly different from the $\beta=0$ case. Comparisons of figure $3(a-d)$ with figure 2(a), and figure 4(a-d) with figure $2(b)$ shows the dominating role of $W_{\beta}^{u}$ in the time-evolution of the scalar field. We note that for the parameter values considered here the size of KAM islands is negligible and, as far as the distribution of the scalar field is concerned, $W_{\beta}^{u}(\bar{\phi})$ is the main organizing structure over the entire domain of the flow with the exception of a narrow near-wall boundary layer, as is evident from figures 3,4 . 
(a)

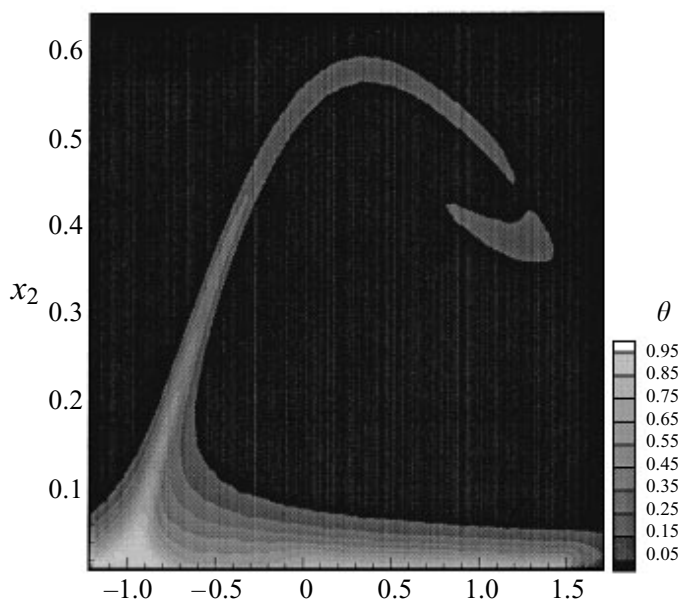

(c)

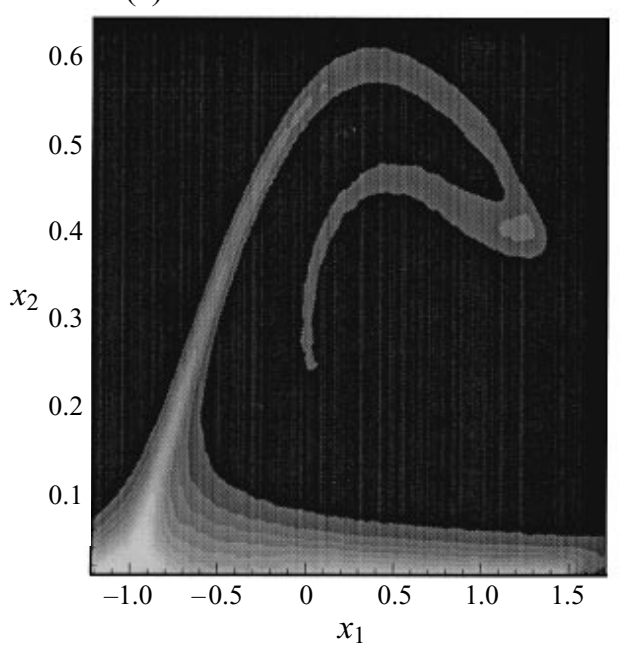

(b)

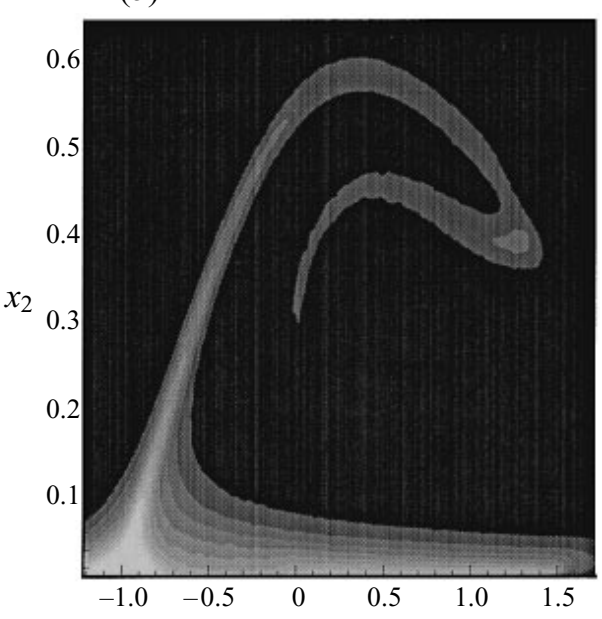

(d)

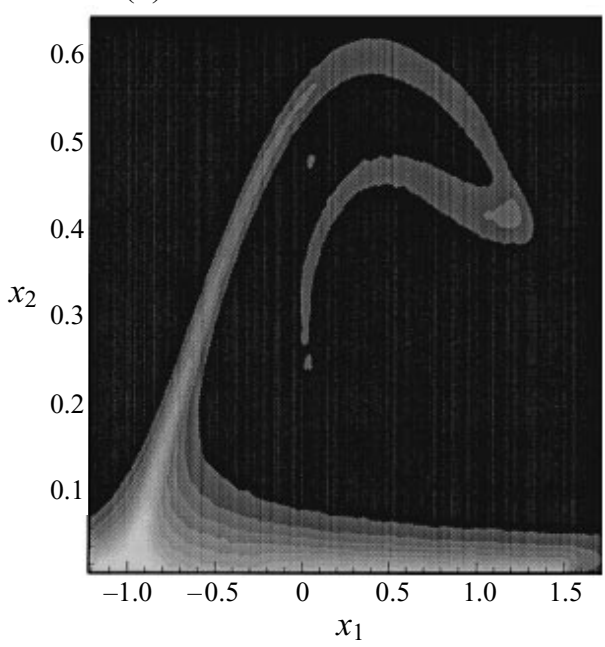

FIgURE 3. Scalar field for $\beta=0.6, \omega=0.72, P e=2.5 \times 10^{4}$, at $(a) t=5 T$, (b) $t=10 T$, (c) $t=20 T,(d) t=30 T, T=2 \pi / \omega$.

Notice further that the distribution of the scalar field is more uniform and widespread for the near-integrable system (figure 4) in contrast to the case with larger $\beta$ (figure 3). Comparing figure 3 with figure 4 , it is also evident that at larger $\beta$ an asymptotic distribution is attained in shorter time. To further pursue this point, the perturbation amplitude in the chaotic advection field is pushed higher to $\beta=0.8$ while retaining $\omega=0.72$, and the scalar field is displayed in figure $6(a-d)$. The scalar field is almost entirely localized around the unstable manifold of $p^{-}$and an asymptotic distribution is attained in even shorter time. We make a few remarks: chaotic advection is usually associated with improved stirring (Aref 1984; Khakhar \& Ottino 1986) and, consequently, improved mixing (Jones 1991). For chaotic advection in bounded domains this results in rapid homogenization of the scalar field, irrespective of the initial distribution; the asymptotic distribution is uniform and the time-scale for homogenization goes as the logarithm (Jones 1991) of the Péclet number, in contrast 
(a)

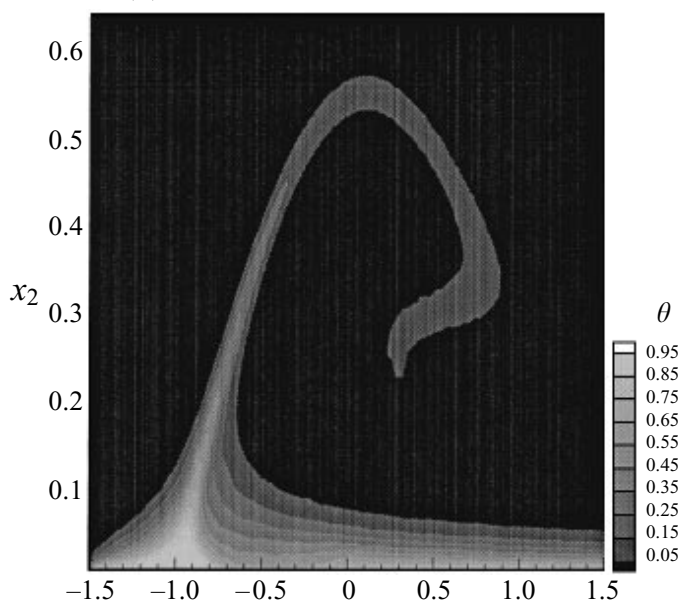

(c)

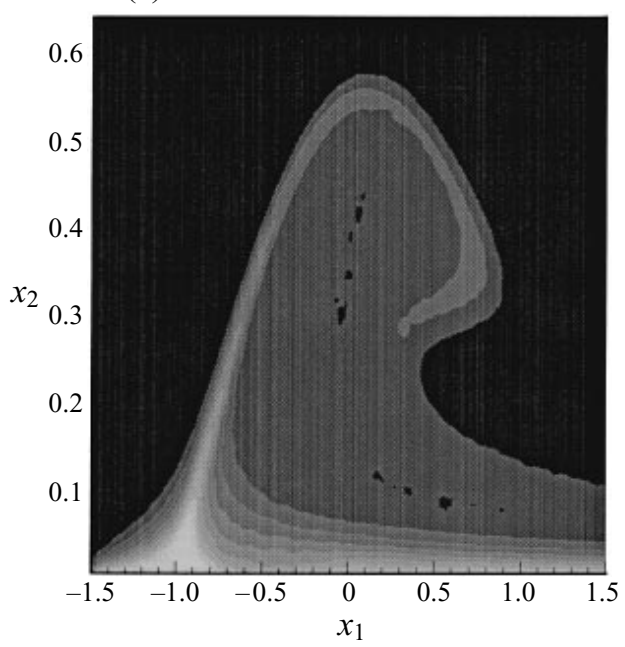

(b)

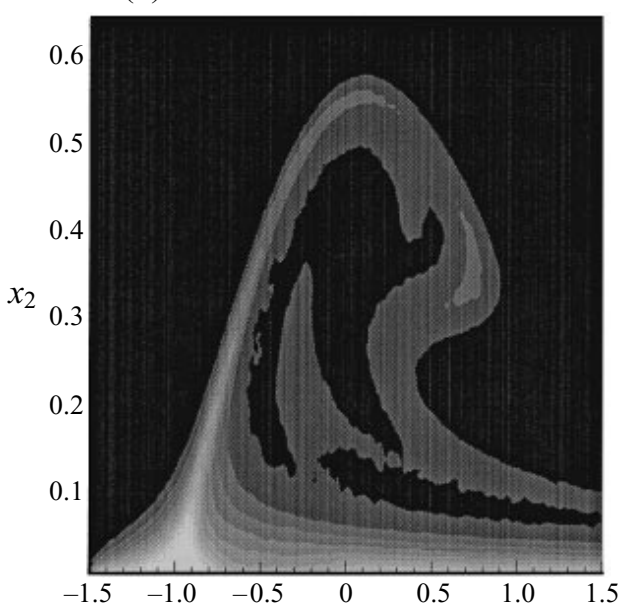

(d)

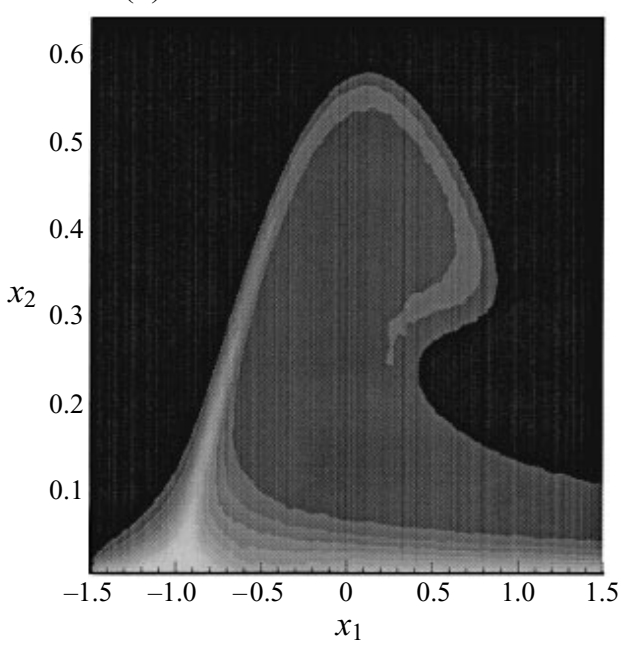

Figure 4. Scalar field for $\beta=0.2, \omega=0.72, P e=2.5 \times 10^{4}$, at $(a) t=5 T,(b) t=10 T$, (c) $t=20 T,(d) t=30 T, T=2 \pi / \omega$.

to the case when particle trajectories are regular and the time-scale of homogenization is linear (Rhines \& Young 1983) in Pe. However, because of the unbounded nature of the flow being considered here, the scalar distribution of course never attains a homogenized state. Indeed, as the chaotic particle motion becomes more widespread (i.e. at larger $\beta$ ) the scalar distribution becomes more localized and non-uniform. Therefore, while the time-scale of homogenization is a useful measure of efficiency of mixing in bounded domains, in unbounded flows there is no analogous physical basis for defining an efficiency of mixing. Below we consider the case with surviving KAM tori enclosing the core region of the bubble.

Increasing the frequency $\omega$ of the perturbation dramatically changes the structure of the unstable manifold $W_{\beta}^{u}$, which now does not sweep out the entire bubble region. Appearing in the core region of the bubble are KAM tori and island bands which are impenetrable by the unstable manifold, and $W_{\beta}^{u}$ is no longer the dominant organizing 
(a)

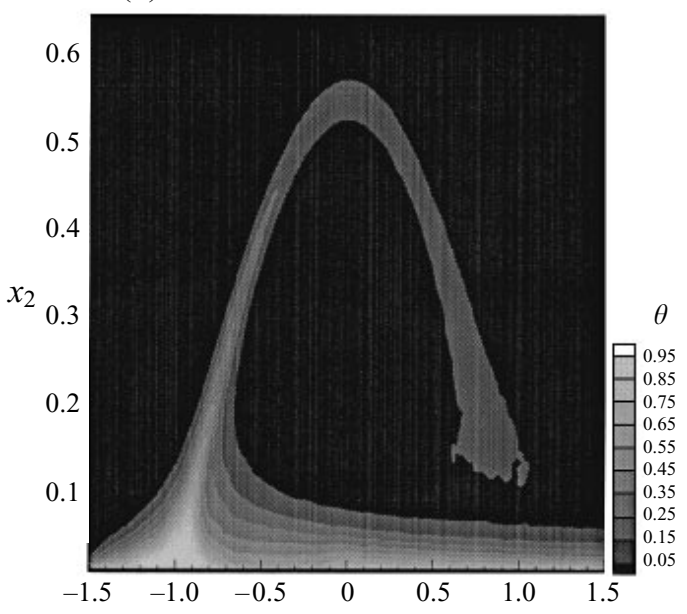

(c)

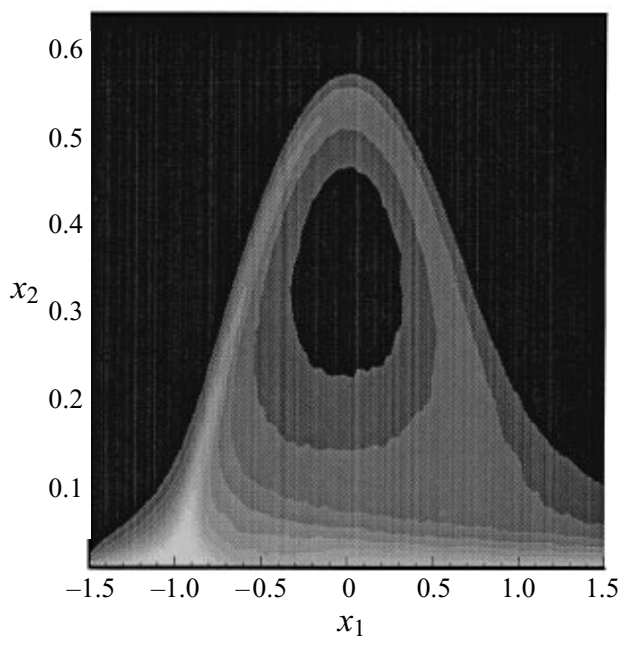

(b)

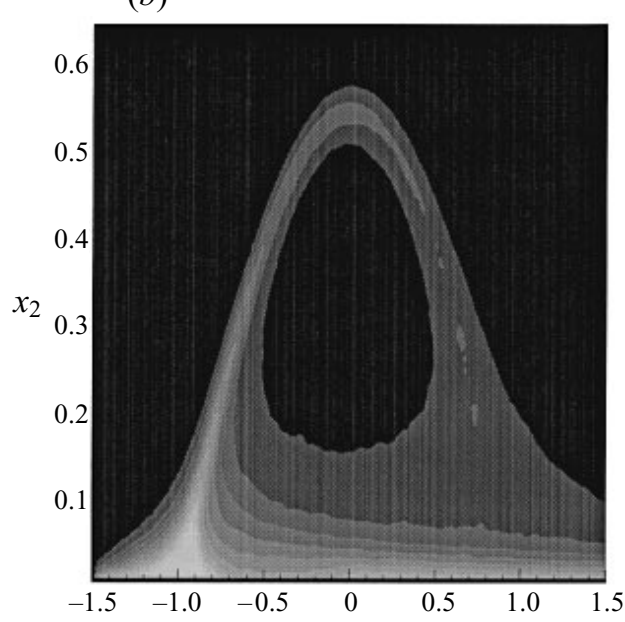

$(d)$

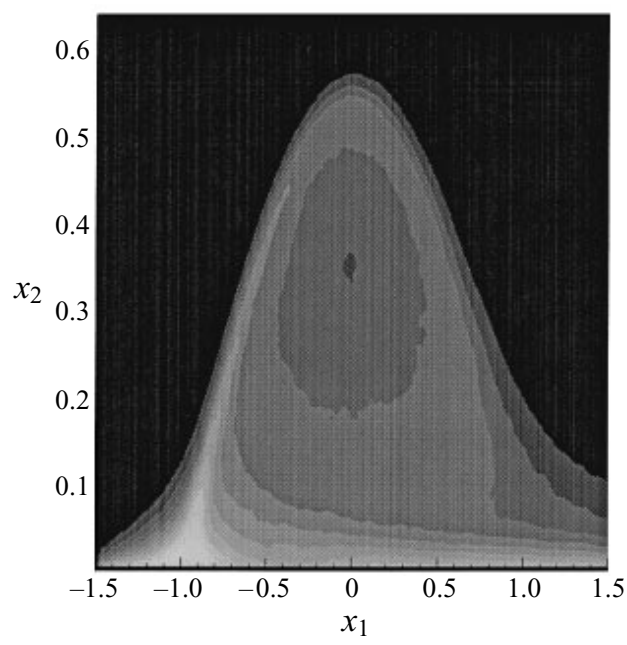

FIGURE 5. Scalar field for the steady separation bubble, for $P e=2.5 \times 10^{4}$, at (a) $t=5 T$, (b) $t=10 T,(c) t=20 T,(d) t=60 T, T$ same as in figures 3,4 .

structure in the time-evolution of the scalar field, though it does influence the scalar distribution in regions swept by $W_{\beta}^{u}$. In figure 7 a Poincare map of the time-periodic chaotic advection field is obtained at $\beta=0.2, \omega=2.0$, showing the structure of the KAM tori and island bands. In figure 8 we compute the scalar field at $\beta=0.2$ and perturbation frequency increased to $\omega=2.0$, while $P e$ is again taken to be $2.5 \times 10^{4}$; to compare with previous results, the time-dependent distribution is obtained at the same times as before. Notice that we are no longer evaluating the scalar field at times that are integral multiples of the perturbation period corresponding to $\omega=2.0$, and therefore the $t$ in figure 8 correspond to different cross-sections $\bar{\phi}$; as $\bar{\phi}$ changes, so does the geometrical structure of $W_{\beta}^{u}(\bar{\phi})$, which in turn influences the distribution of the passive scalar in regions swept by $W_{\beta}^{u}(\bar{\phi})$. But in the core region of the bubble the KAM tori play a role not unlike closed streamlines in the steady bubble as transport 
(a)

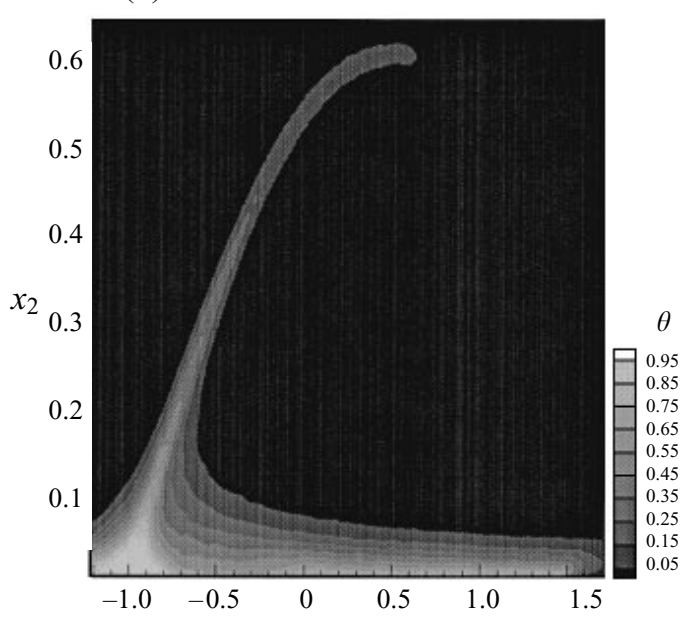

(c)

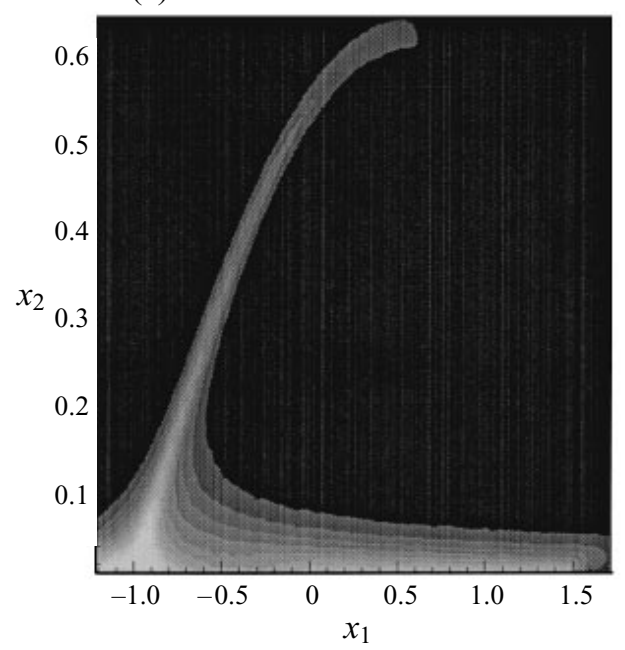

(b)

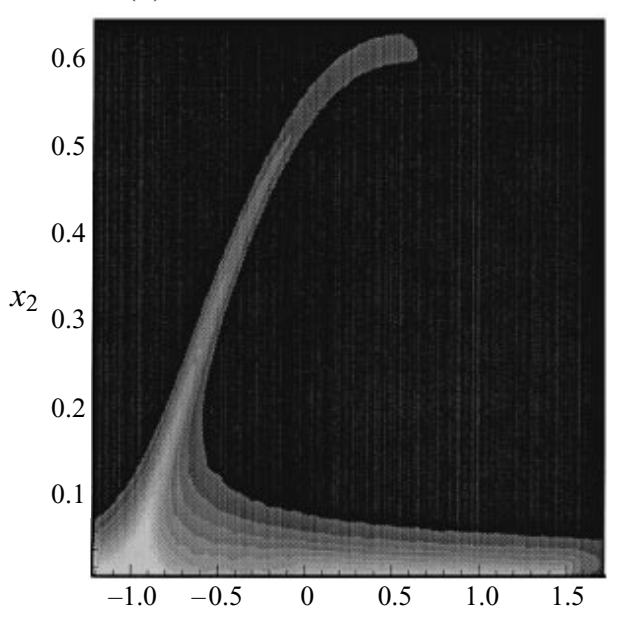

(d)

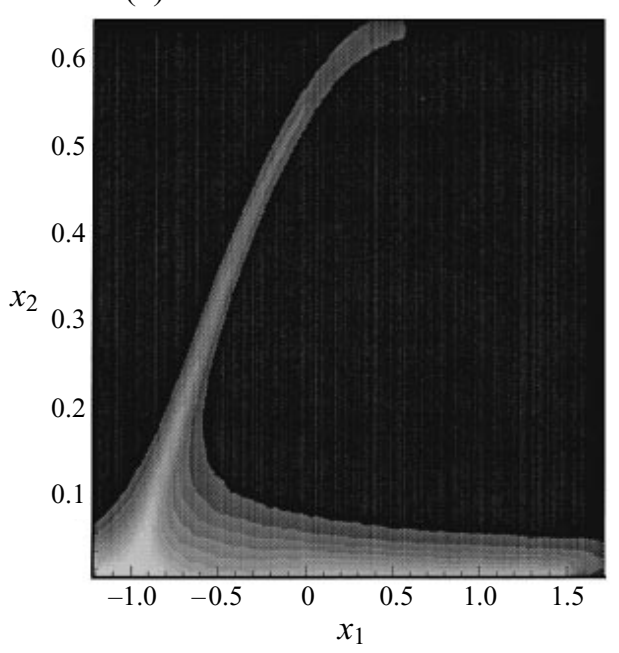

FIgURE 6. Scalar field for $\beta=0.8, \omega=0.72, P e=2.5 \times 10^{4}$, at $(a) t=5 T$, (b) $t=10 T$, (c) $t=20 T,(d) t=30 T, T=2 \pi / \omega$.

across a KAM torus is possible by scalar diffusion only, and the time-evolution of the scalar field in this core region resembles closely that in the steady-bubble case. Nothing can be said though about the nature of the asymptotic distribution in this case.

We conclude this section with a verification of the results obtained from the whole field computations, using the Wiener bundle method. Centreline profiles of the scalar field along $\left(x_{1}=0, x_{2}>0\right)$ were extracted from figure $3(a, d)$, and these are plotted as solid lines in figure 9. Point values of the scalar field were obtained at the same $t$ using the Wiener bundle method and these are also displayed in figure 9. The scalar value at a point on the centreline is obtained by integrating backwards in time a collection $N$ of 'particles' that are located at the given $x \equiv\left(x_{1}=0, x_{2}>0\right)$ at time $t$, using the stochastic differential of (3.3). As was mentioned earlier, the wall can 


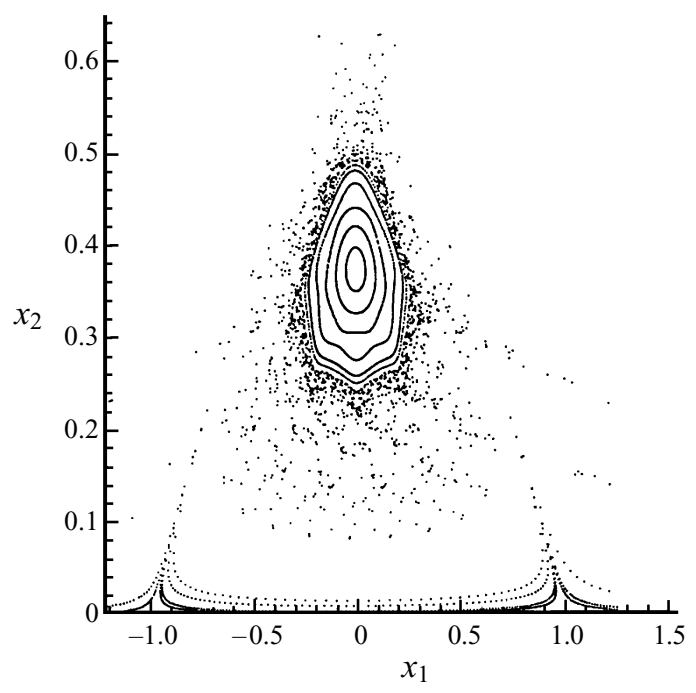

Figure 7. A Poincaré map of the bubble region for $\beta=0.2, \omega=2.0$.

(a)

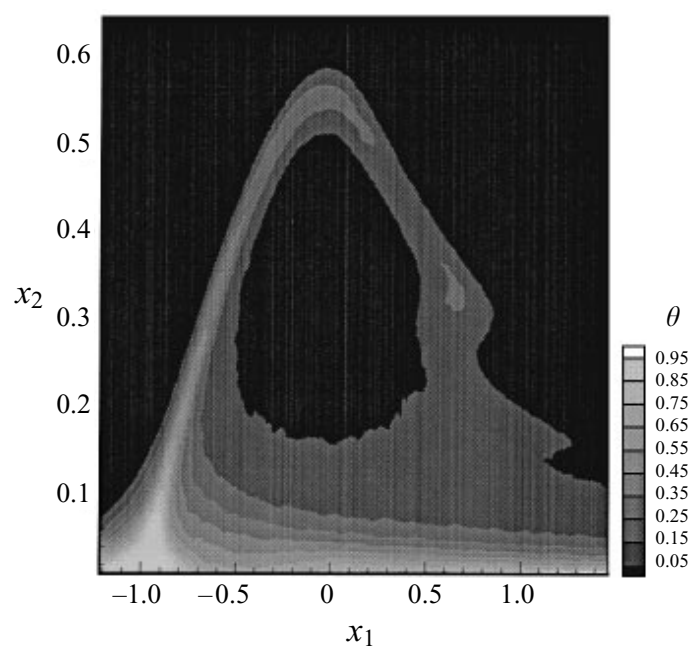

(b)

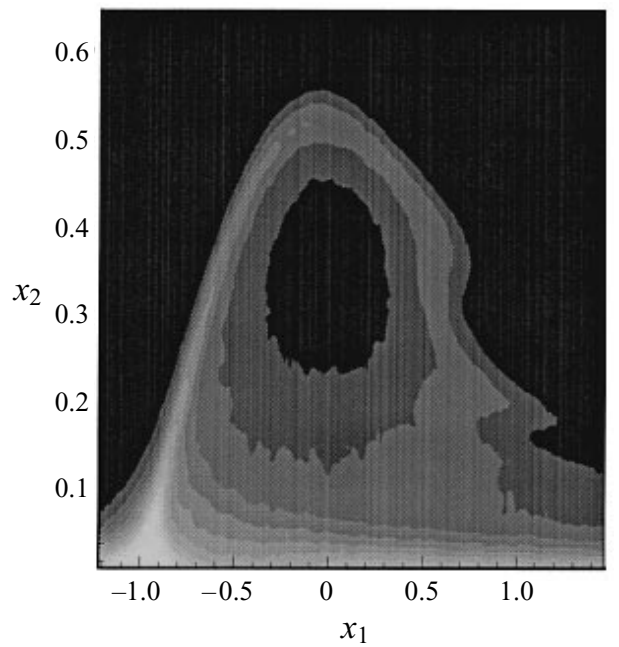

FIgURE 8. Scalar field for $\beta=0.2, \omega=2.0, P e=2.5 \times 10^{4}$, at $(a) t=10 T,(b) t=20 T$, where $T$ is same as in figures $3-6$, i.e. $T=2 \pi / 0.72$.

be treated as a perfectly absorbing boundary for a Brownian particle and Wiener trajectories are terminated on reaching the wall in backward time. The scalar value $\theta(x, t)$ at $x \equiv\left(x_{1}, x_{2}>0\right)$ at time $t$ is given by

$$
\theta=\frac{1}{N} \sum_{i=1}^{N} \delta_{w i}
$$

where $\delta_{w i}=1$ if the $i$ th particle reaches the active portion of the wall before time $t$, and $\delta_{w i}=0$ otherwise. Equation (3.6) is therefore an expectation over the scalar values 'carried' by the Wiener trajectories that arrive at the point $x$ in time $t$. Standard error estimates (Ghosh 1994) show the error is $O\left(N^{-1 / 2}\right) ; N$ was taken to be $2 \times 10^{4}$ 

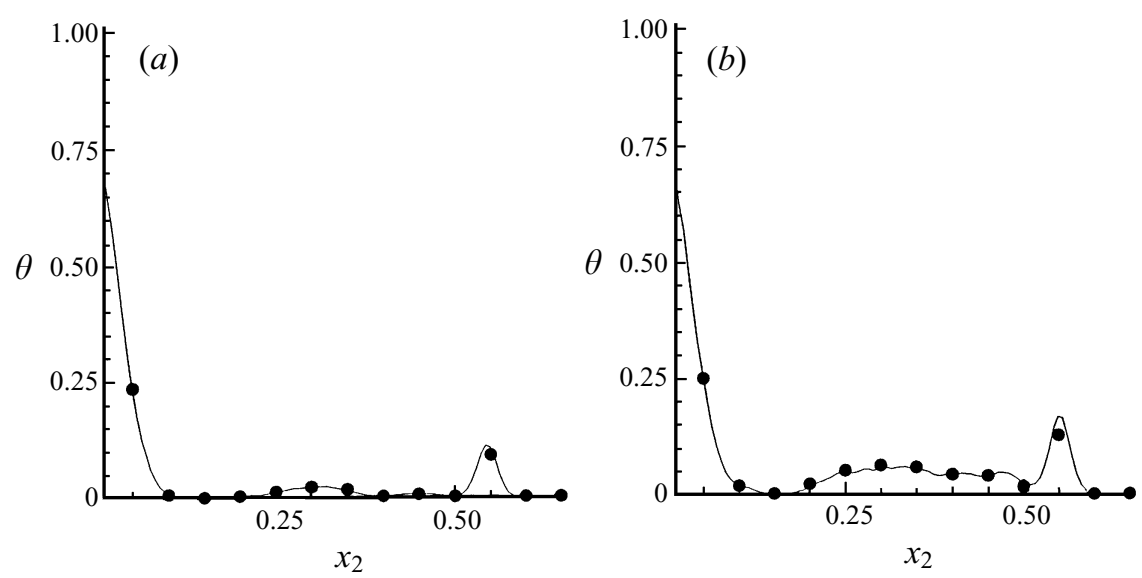

FIGURE 9. Distribution of the scalar field along the centreline $\left(x_{1}=0, x_{2}>0\right)$, extracted from figure 3 , at $(a) t=5 T,(b) t=30 T$, shown in solid lines; $T$ same as in figure 3 . Dots represent Wiener bundle solutions at $\beta=0.6, \omega=0.72, P e=2.5 \times 10^{4}$.

in our computations. From figure 9 it is evident that the match between the results obtained using the two independent random-walk schemes is quite satisfactory.

\subsection{Flux of passive scalar from the local active surface into the thermal boundary layer}

The flux at the wall, integrated over the active portion of the wall and averaged over time $T=2 \pi / \omega$, is evaluated at every $t=m \frac{1}{2} T, m$ an odd integer, for $\beta$ equal to 0 , 0.2 , and 0.6 , and $\omega=0.72$, and the results are displayed in figure $10(a)$. It is evident that the time-averaged wall-integrated flux approaches an asymptotic value over a time-scale that appears to be unrelated to the time-scales over which the scalar field attains an asymptotic distribution away from the wall.

Moreover, the chaotic particle motion increases the asymptotic flux at the wall only marginally over the steady-advection case $(\beta=0)$. The asymptotic value of the time-averaged wall-integrated flux is evaluated at several perturbation amplitudes, $\beta$ ranging from zero to unity (keeping $\omega$ and $P e$ fixed), and these are displayed in figure $10(b)$. It is clear that there is little or no enhancement over the $\beta=0$ case. Both observations are closely related to the fact that the dynamics of particle motion in the near-wall region, as well as the boundary layer distribution of the scalar field, are largely insensitive to the chaotic advection-diffusion process away from the wall. It is also observed that the asymptotic value of $\left\langle J_{w}(t)\right\rangle_{T}$ appears to be independent of the averaging period $T$, though averaging over shorter times does increase the scatter in the data, which is only to be expected in a random-walk computation.

These observations can be explained by considering the relative contributions of the various terms in the advection-diffusion equation of (3.1) in the near-wall thermal boundary layer, once the initial transients have died down and the scalar field has settled into an asymptotic state. We focus our attention on the near-wall thermal boundary layer at $x_{1} \in(-1.5,-1) \cup(-1,1.5)$; we exclude a small neighbourhood of the separation point $p^{-}$, where the boundary layer approximation is invalid (Acrivos $\&$ Goddard 1965 ). Using a scaled time variable $\tau=\omega t$ and the boundary layer coordinate $\hat{x}_{2}=P e^{1 / 3} x_{2}$, the energy equation of (3.1) with advection field $u$ given by 

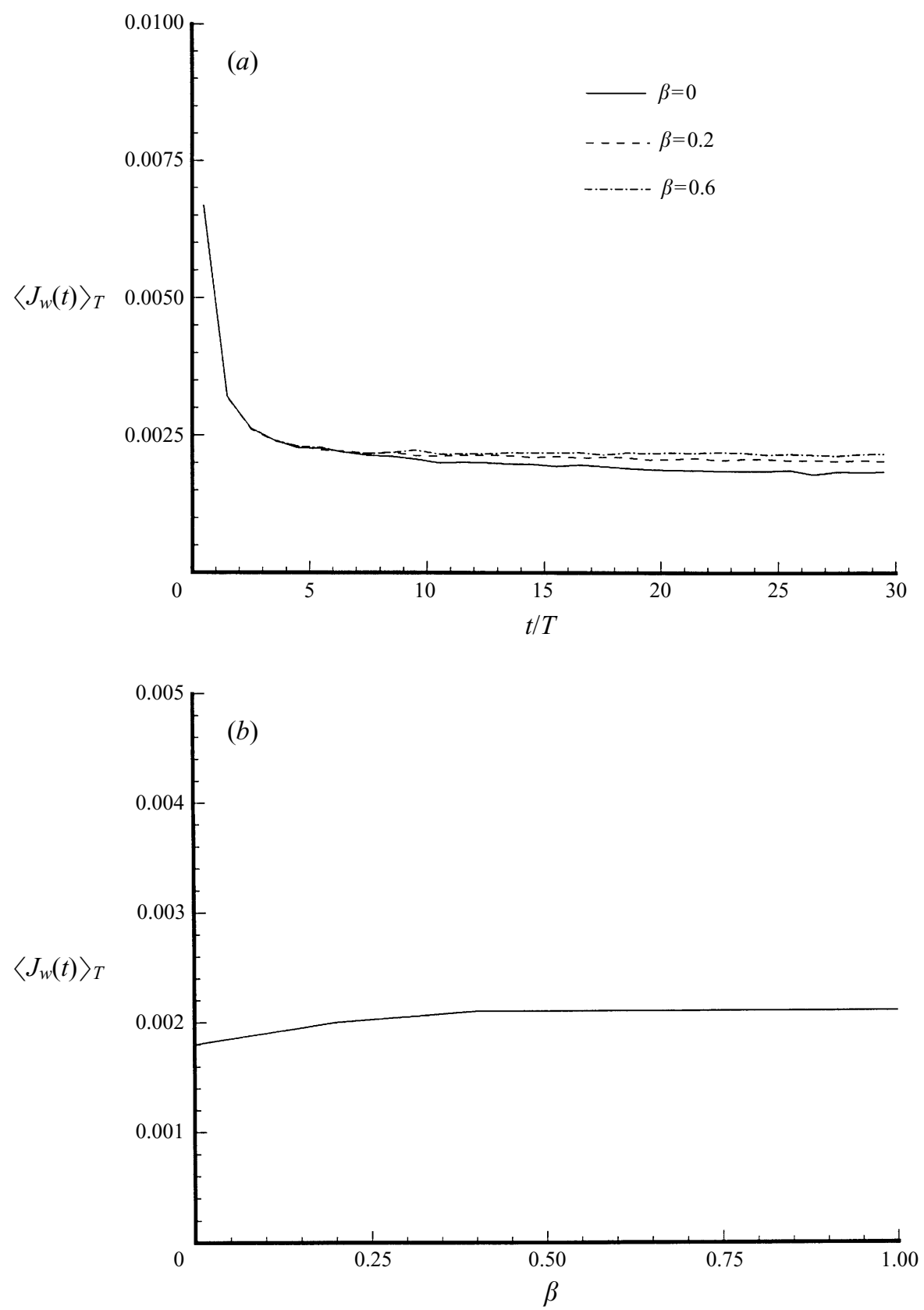

FIgURE 10. Time-averaged wall-integrated flux $\left\langle J_{w}(t)\right\rangle_{T}$ for $\beta=0,0.2$ and 0.6 and asymptotic $\left\langle J_{w}(t)\right\rangle_{T}$ at several $\beta \in[0,1]$, for $\omega=0.72, P e=2.5 \times 10^{4}$, and $T=2 \pi / \omega$.

(2.8) becomes

$$
\eta \frac{\partial \theta}{\partial \tau}+\left(x_{1}^{2}-1\right) \hat{x}_{2} \frac{\partial \theta}{\partial x_{1}}-x_{1} \hat{x}_{2}^{2} \frac{\partial \theta}{\partial \hat{x}_{2}}-\frac{\partial^{2} \theta}{\partial \hat{x}_{2}^{2}}+O\left(P e^{-1 / 3}\right)=0,
$$

where $\eta=\omega / P e^{-1 / 3}$. For large $\eta$, (3.7) is clearly a singular perturbation problem. We shall restrict attention to the following subcase of $\eta \rightarrow \infty: \omega$ fixed and $P e \rightarrow \infty$. 
Introducing the small parameter $\gamma=\eta^{-1 / 2}$ in (3.7) gives

$$
\frac{\partial \theta}{\partial \tau}+\gamma^{2}\left[\left(x_{1}^{2}-1\right) \hat{x}_{2} \frac{\partial \theta}{\partial x_{1}}-x_{1} \hat{x}_{2}^{2} \frac{\partial \theta}{\partial \hat{x}_{2}}\right]=\gamma^{2} \frac{\partial^{2} \theta}{\partial \hat{x}_{2}^{2}}+O\left(\gamma^{4}\right) .
$$

In an inner boundary layer of thickness $(\omega P e)^{-1 / 2}$, the first and third terms of (3.8) will be in balance. We shall seek asymptotic solutions in the outer and inner regions in the form of a power series in $\gamma$. Typically, these solutions would be linked by the method of matched asymptotic solutions. However, we shall only look at the form of the solutions in the outer and inner regions and the matching conditions between the two solutions in order to extract useful order estimates. A full asymptotically matched solution will not be attempted becuse of the difficulty in applying a matching condition at the separation point $p^{-}$(at $\left.x_{1}=-1\right)$, linking the solution in the thermal boundary layer with the thermal wake along the unstable manifold.

With $\theta=\hat{\theta}\left(\tau, x_{1}, \hat{x}_{2}\right)$ denoting the outer representation, we substitute the ansatz

$$
\hat{\theta}=\sum_{n=0}^{\infty} \gamma^{n} \hat{\theta}_{n}
$$

in (3.8) and on equating like powers of $\gamma$, we obtain for the first five terms:

$$
\frac{\partial \hat{\theta}_{n}}{\partial \tau}= \begin{cases}0, & n=0,1 \\ \Xi\left(n, x_{1}, \hat{x}_{2}\right), & n=2,3 \\ \Xi\left(n, x_{1}, \hat{x}_{2}\right)-3 \omega \hat{x}_{2}{ }^{2}\left(1+x_{1} \beta \sin \tau\right) \frac{\partial \hat{\theta}_{0}}{\partial x_{1}}+\omega \hat{x}_{2}{ }^{3} \beta \sin (\tau) \frac{\partial \hat{\theta}_{0}}{\partial \hat{x}_{2}}, & n=4\end{cases}
$$

where

$$
\Xi\left(n, x_{1}, \hat{x}_{2}\right)=\frac{\partial^{2} \hat{\theta}_{(n-2)}}{\partial \hat{x}_{2}{ }^{2}}-\left(x_{1}{ }^{2}-1\right) \hat{x}_{2} \frac{\partial \hat{\theta}_{(n-2)}}{\partial x_{1}}+x_{1} \hat{x}_{2}{ }^{2} \frac{\partial \hat{\theta}_{(n-2)}}{\partial \hat{x}_{2}} .
$$

We expect steady-state oscillations in the temperature field in the near-wall thermal boundary layer owing to the steady-state oscillations in the local wall shear (Pedley 1972); this is confirmed by the numerically obtained temperature time-series in figure 11(a). Thus we cannot allow secular terms in the temperature field that would grow indefinitely with time. Inspecting the first five terms in the expansion given above, we find that no time-periodic solutions are possible for the first four terms of (3.9), and therefore require

$$
\frac{\partial \hat{\theta}_{n}}{\partial \tau}=0, \quad n<4 .
$$

Then the time-dependent component of (3.9) is (at most) $O\left(\gamma^{4}\right)$ which, in terms of the Péclet number, is $O\left(P e^{-2 / 3}\right)$. We note that for the Péclet number at which the temperature time-series of figure 11(a) was obtained, $P e^{-1 / 3}=0.03$; since figure 11(a) was obtained at $\left(x_{1}, x_{2}\right)=(0,0.04)$, the result of figure $11(a)$ can be considered to be representative of the temperature field in the outer region. We also confirm the weak $P e^{-2 / 3}$ scaling of the steady-state temperature oscillations by repeating the numerical computation of figure $11(a)$ at two different Péclet numbers, and the results are displayed in figure $11(b)$.

We mention in passing that the asymptotic analysis, of course, does not apply away from the wall and the asymptotic scalar distribution is not necessarily timeindependent, even to dominant order. Time-series obtained at a point in the bubble 

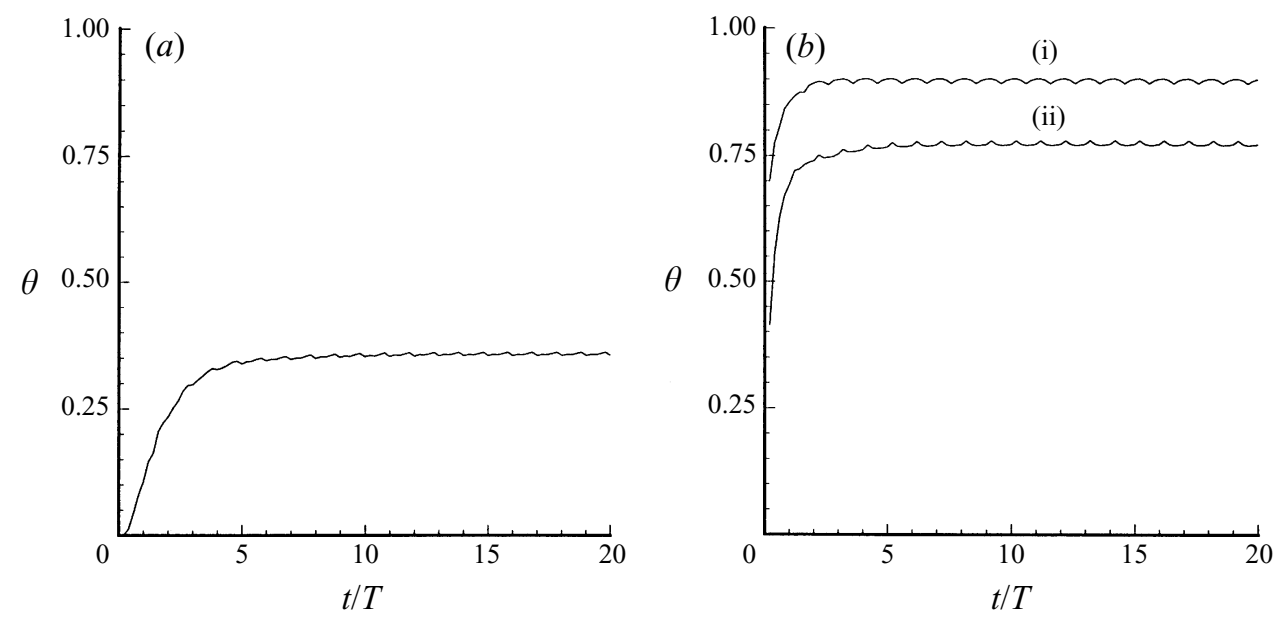

FIGURE 11. Time-series using the Wiener bundle method, at $\left(x_{1}, x_{2}\right)=(0,0.04)$, for $\beta=0.6$, $\omega=0.72$ and (a) $P e=2.5 \times 10^{4}$; (b) $P e=125$ (i), $P e=10^{3}$ (ii).

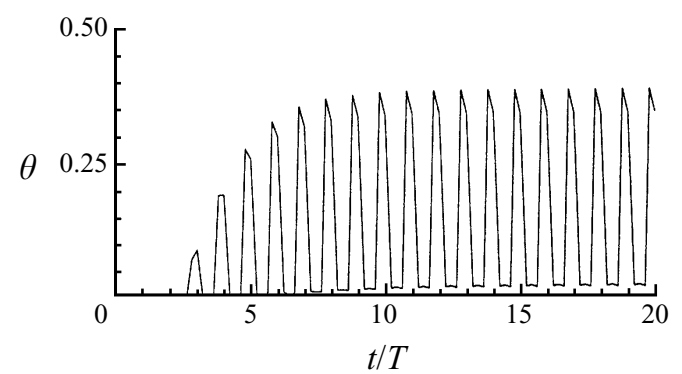

FIgURE 12. Time-series using the Wiener bundle method, at $\left(x_{1}, x_{2}\right)=(-0.547,0.321) \in W_{\beta}^{u}$, for $\beta=0.6, \omega=0.72, P e=2.5 \times 10^{4}$.

region away from the wall (see figure 12) shows large $O(1)$ time-periodic fluctuations about an asymptotic mean value, in sharp contrast to figure 11(a).

To dominant order, the instantaneous wall-integrated flux is given by

$$
J_{w}(t)=\left.P e^{-2 / 3} \int_{-1.5}^{1.5} \frac{\partial \theta}{\partial \hat{x}_{2}}\right|_{\hat{x}_{2} \rightarrow 0} \mathrm{~d} x_{1} .
$$

It can be shown (Ghosh 1994) that the contribution to the instantaneous wallintegrated flux from the thermal boundary layer region in the vicinity of the separation point $p^{-}$can be ignored to dominant order, and therefore does not appear in the expression above.

To obtain estimates of the heat flux from the wall, it is essential to consider the inner region. Let $\xi=\gamma^{-1} \hat{x}_{2}$ be a suitably scaled inner variable. With $\theta=\Theta\left(\tau, x_{1}, \xi\right)$ denoting the inner representation, we substitute the ansatz

$$
\Theta=\sum_{n=0}^{\infty} \gamma^{n} \Theta_{n}
$$

in (3.8) and on equating like powers of $\gamma$, we obtain a transient diffusion equation for 
the first two terms:

$$
\frac{\partial \Theta_{n}}{\partial \tau}-\frac{\partial^{2} \Theta_{n}}{\partial \xi^{2}}=0, \quad n=0,1 .
$$

'Diffusing solutions' of (3.10), which are solutions in terms of the similarity variable $\xi^{2} / \tau$, are ruled out since they apply only to the transient state and not the asymptotic steady-state oscillations that are of interest here. Then, a consideration of the asymptotic matching condition at $\xi \rightarrow \infty$ yields easily

$$
\Theta_{0}=1, \Theta_{1}=C \xi,
$$

where use was made of the fact that $\hat{\theta}_{0}\left(x_{1}, \hat{x}_{2} \rightarrow 0\right)$ must be of the form $1+a_{0} h(\beta)$ with $h(\beta) \rightarrow 0$ as $\beta \rightarrow 0$, since the outer solution must approach the steady solution at all $\hat{x}_{2}$ and $\gamma$ as the amplitude $\beta$ of the time-periodic perturbation approaches zero; rejecting the diffusing solution at zeroth order requires $a_{0}=0$, while the constant $C$ in (3.11) is obtained from the asymptotic matching at $\xi \rightarrow \infty$ (see Pedley 1972). From (3.11) we obtain easily that the wall-integrated flux is $O\left(\mathrm{Pe}^{-2 / 3}\right)$, identical to the steady case. The marginal enhancement observed in figure $10(b)$ is interpreted as a weak second-order effect. We also note that the asymptotic instantaneous wall-integrated flux, $J_{w}(t)$, is time-independent to dominant order, which accounts for the observed feature that the asymptotic time-averaged wall integrated flux, $\left\langle J_{w}(t)\right\rangle_{T}$, is largely insensitive to the averaging period $\mathrm{T}$.

In physical terms, the introduction of a time-periodic perturbation to the advection field can cause an enhancement in the heat flux from the wall in two ways: one due to the oscillating wall shear, and another due to the chaotic advection in the bubble region above the thermal boundary layer, which causes the destruction of the separatrix boundary layer and the constant temperature recirculation bubble, allowing heat to escape more rapidly from the boundary layer into the overlying bubble region. But as the analysis above shows, neither of these is a dominant order effect. Their influence at lower orders is not captured by our analysis above.

\subsection{Case with oscillating points of zero shear stress}

Results obtained by Shariff et al. (1991) show that for two-dimensional time-periodic incompressible flows adjacent to a plane no-slip boundary, the necessary condition for a point on the no-slip boundary $\left(x_{1}^{*}, 0\right)$ to have a one-dimensional stable/unstable manifold for the corresponding Poincare map, with the manifold emanating with non-zero slope, is the following:

$$
\int_{0}^{T} \frac{\partial u_{1}}{\partial x_{2}}\left(x_{1}=x_{1}^{*}, x_{2}=0\right) \mathrm{d} t=0
$$

where $x_{1}$ is the coordinate along the boundary, $x_{2}$ is the coordinate normal to the boundary, and $u_{1}$ is the $x_{1}$-component of the velocity field, i.e. the time-averaged shear stress must vanish at the manifold emanation points. Here we point out its consequences on the scalar distribution in the context of our advection-diffusion problem.

To this end, we first set-up a chaotic advection field which has oscillating points of zero shear stress on the no-slip boundary. The time-periodic velocity field of (2.8) has two points of zero shear stress on the no-slip boundary at all times, given by $x_{1}= \pm 1$, i.e. the points of zero shear stress are independent of time $t$. The point $(-1,0)$ corresponds to the separation point $p^{-}$, while $(+1,0)$ corresponds to the attachment point $p^{+}$. For the corresponding Poincaré map $P_{\beta}^{\bar{\phi}}, p^{-}$and $p^{+}$are the only points on 
the no-slip boundary having one-dimensional unstable/stable manifolds, irrespective of the cross-section $\bar{\phi}$. Consider now a time-periodic perturbation of (2.5) that not only generates chaotic particle trajectories in the bubble region but also creates two points of zero shear stress on the no-slip boundary that are not time-independent, but instead oscillate periodically about $x_{1}= \pm 1$. This is achieved easily by adding an appropriate perturbation term to (2.3); the boundary vorticity in the time-dependent flow is made to vary according to the equation

$$
w\left(x_{2}=0\right) \equiv-\left.\frac{\partial u_{1}}{\partial x_{2}}\right|_{x_{2}=0}=-K\left(x_{1}^{2}-x_{s}^{2}+\beta x_{1} x_{s} \sin (\omega t)\right) .
$$

The appropriately non-dimensionalized velocity field, truncated again to third-order, is given by

$$
\left.\begin{array}{l}
u_{1}=-x_{2}+3 x_{2}^{2}+x_{1}^{2} x_{2}-\frac{2}{3} x_{2}^{3}+\beta x_{1} x_{2} \sin (\omega t), \\
u_{2}=-x_{1} x_{2}^{2}-\frac{\beta}{2} x_{2}^{2} \sin (\omega t),
\end{array}\right\}
$$

which is again an asymptotically exact solution of the Navier-Stokes and continuity equations close to the origin of the expansion. The points of zero shear stress on the no-slip boundary $\left(x_{1}, 0\right)$ are now given by

$$
x_{1}= \pm 1-\frac{\beta}{2} \sin (\omega t)+O\left(\beta^{2}\right) .
$$

However, applying Shariff et al.'s result of (3.12), for the Poincare map $P_{\beta}^{\bar{\phi}}$ corresponding to the velocity field of (3.13) the only points on the no-slip boundary $\left(x_{1}^{*}, 0\right)$ having a one-dimensional stable/unstable manifold are located at $x_{1}^{*}= \pm 1$. Notice that the necessary condition of (3.12) is independent of the cross-section or phase $\bar{\phi}$ of the Poincare map $P_{\beta}^{\bar{\phi}}$, which implies that though the points of zero shear stress on the no-slip boundary oscillate in a time-periodic manner, the manifold emanation points are time-independent and remain the same for every $P_{\beta}^{\bar{\phi}}$, irrespective of $\bar{\phi}$. Therefore, while the unstable manifold $W_{\beta}^{u}(\bar{\phi})$ changes structure with $\bar{\phi}$, the manifold emanation point or separation point remains stationary at the wall. This property becomes transparent from numerical simulation results of figure $13(a-c)$ for the scalar distribution computed at times that are not integral multiples of the period $T=2 \pi / \omega$ of the timeperiodic velocity field of (3.13) and therefore correspond to different cross-sections $\bar{\phi}$. Thus, despite the fact that (3.13) and (2.8) are very different chaotic advection fields, the scalar distributions for the associated advection-diffusion problem at large Péclet numbers share the same qualitative features owing to the similarity in the underlying geometrical structures in the two cases. This further reinforces the importance of the underlying geometrical structures in determining the scalar distribution in chaotic advection fields.

The asymptotic time-averaged wall-integrated flux $\left\langle J_{w}(t)\right\rangle_{T}$ again shows weak enhancement over the steady-advection case, which is not surprising following our discussion in $\$ 3.4$. Temperature time-series in the thermal boundary layer indicate weak time-dependence as before, attributed to the absence of time-dependent structures in the near-wall region in the sense of oscillating manifold emanation points. The absence of such time-dependent structures is the likely cause of the lack of enhanced transport usually associated with turbulent flows. Moreover, it should be clear from equation (3.12) that every time-periodic two-dimensional velocity field will have timeindependent manifold emanation points at the no-slip boundary. Finally, we note that 
(a)

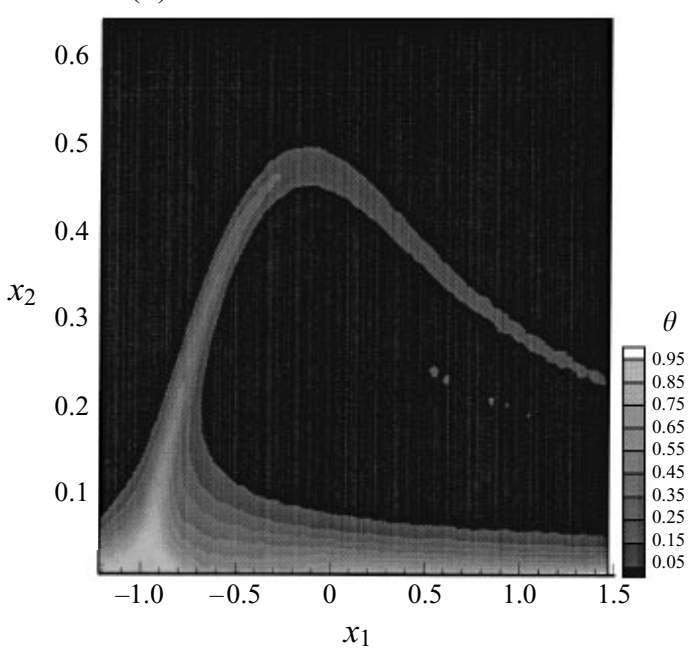

(b)

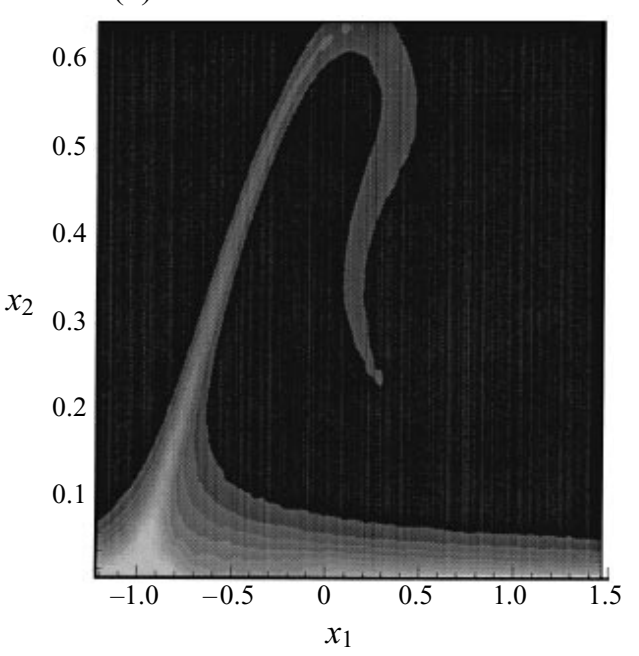

(c)

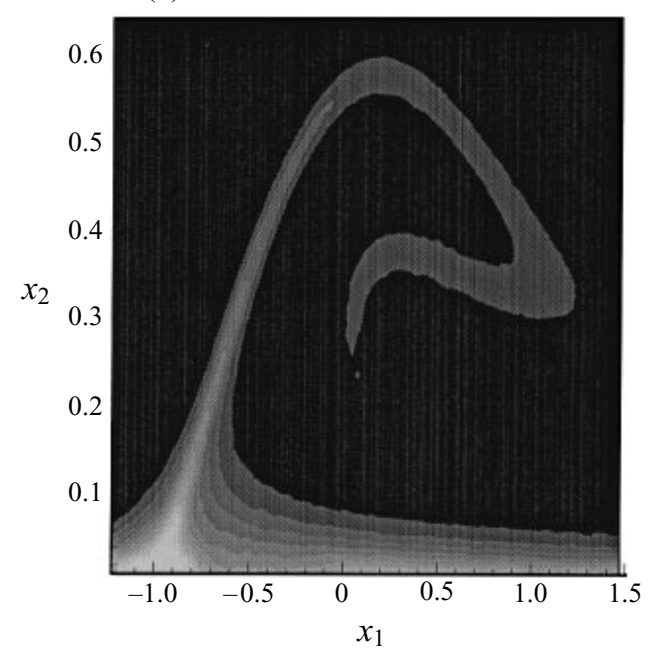

FigURE 13. Scalar field corresponding to the chaotic advection field of (3.13), for $\beta=0.4, \omega=0.65$, $P e=2.5 \times 10^{4}$, at $(a) t=5.25 T,(b) t=5.75 T,(c) t=6 T, T=2 \pi / \omega$.

it might be possible to generate time-dependent structures in the near-wall region for two-dimensional chaotic advection fields with more complicated time-dependences, in particular quasi-periodic time-dependence.

\subsection{Impact of changing bubble size}

The size of the steady separation bubble can be varied by varying the coefficient $A_{122}$ in the time-independent velocity field of $(2.4) ; A_{122}$ must lie within the appropriate limits given in $\S 2$. Introducing a time-periodic perturbation as in (2.6) and truncating the series expansion to third-order gives a chaotic advection field identical to (2.8) with time-independent component $f^{u}(x)$, expressed now in terms of the unspecified coefficient $A_{122}$,

$$
f^{u} \equiv\left(f_{1}^{u}, f_{2}^{u}\right)=\left(-x_{2}+\frac{A_{122}}{K x_{s}} x_{2}^{2}+x_{1}^{2} x_{2}-\frac{2}{3} x_{2}^{3},-x_{1} x_{2}^{2}\right) .
$$


(a)

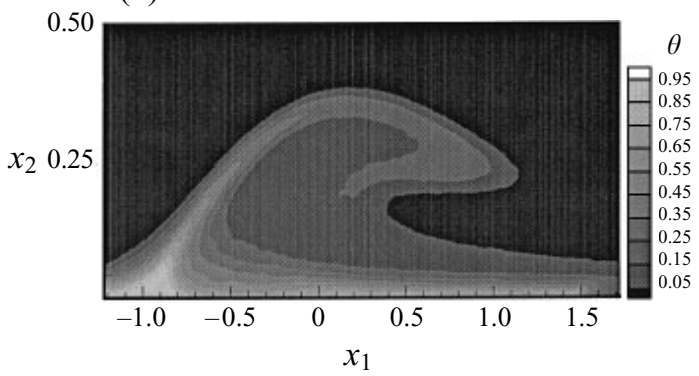

(b)

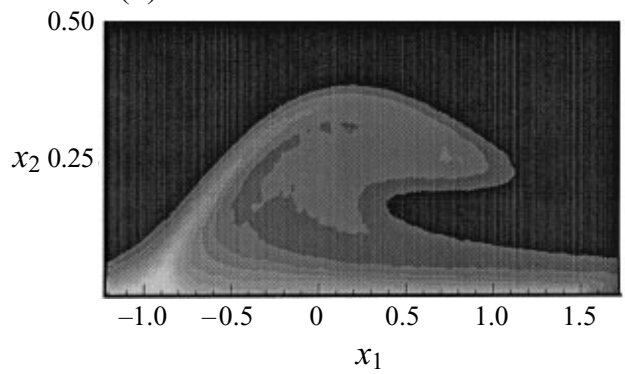

FIGURE 14. Scalar field corresponding to the chaotic advection field with time-independent component (3.14), for $A_{122}=4.5 K x_{s}$, with $\beta=0.5, \omega=0.5, P e=2.5 \times 10^{4}$, at $(a) t=10 T$, (b) $t=20 T$, $T=2 \pi / \omega$.

(a)

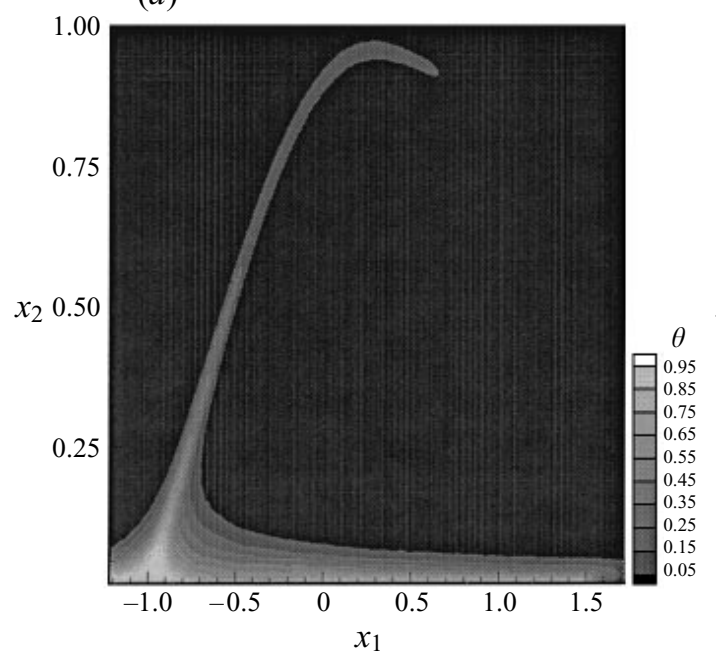

(b)

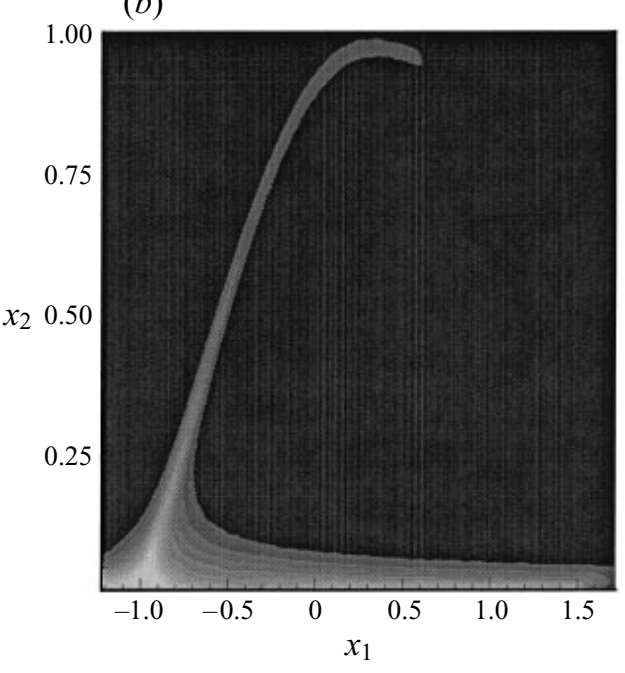

FIGURE 15. Scalar field corresponding to the chaotic advection field with time-independent component (3.14), for $A_{122}=2 K x_{s}$, with $\beta=0.5, \omega=0.5, P e=2.5 \times 10^{4}$, at (a) $t=10 T$, (b) $t=20 T$, $T=2 \pi / \omega$.

We consider here the effect of variations in the coefficient $A_{122}$ on the chaotic advection field above and, consequently, on the scalar distribution in the associated advection-diffusion problem.

Thus far we have presented numerical simulation results for $A_{122}=3 K x_{s}$. Timedependent scalar distributions are now computed at two different values of the coefficient $A_{122}$ : in figure $14(a, b)$, scalar distributions are obtained for $A_{122}=4.5 \mathrm{~K} x_{s}$, while in figure $15(a, b)$ the scalar distributions are obtained for $A_{122}=2 K x_{s}$, with the perturbation parameters $\beta, \omega$ and the Péclet number $P e$ identical in the two cases. Evidently, there is a sharp qualitative difference in the scalar distributions for the two cases. It is observed that at smaller $A_{122}$, the unstable manifold $W_{\beta}^{u}(\bar{\phi})$ plays a more dominant organizing role in the distribution of the passive scalar. Moreover, the distributions are more localized and non-uniform at smaller $A_{122}$. Following our arguments in $\S 3.4$, it is not surprising that the wall-integrated flux is observed to be largely insensitive to variations in $A_{122}$, as long as $A_{122}$ is not too large. 
While for $\beta=0$, i.e. for steady advection, the value of $A_{122}$ determines the size of the separation bubble, for $\beta \neq 0$ but $\beta$ small, $A_{122}$ determines the location of the heteroclinic tangle formed by the intersections of the stable and unstable manifolds, $W_{\beta}^{s}(\bar{\phi})$ and $W_{\beta}^{u}(\bar{\phi})$ respectively, of the Poincare map $P_{\beta}^{\bar{\phi}}$ corresponding to the timeperiodic chaotic advection field. In fact, it is a simple consequence of Gronwall's lemma (e.g. see Wiggins 1990) that trajectories under $P_{\beta}^{\bar{\phi}}$ with initial position on $W_{\beta}^{u}(\bar{\phi})$ within $O(\beta)$ of the separation point $p^{-}$, must remain $O(\beta)$ close to the unperturbed separatrix $\psi_{h}$ for small $\beta$, until they enter a small neighbourhood of the attachment point $p^{+}$. Then, since $A_{122}$ determines the shape of the separatrix $\psi_{h}$, it strongly influences the shape of the unstable manifold $W_{\beta}^{u}(\bar{\phi})$. Moreover, variations in $A_{122}$ cause sharp variations in the stretching properties of the chaotic advection field, evidence of which will be presented later; the lower the value of $A_{122}\left(A_{122}>(8 / 3)^{1 / 2} K x_{s}\right)$, the greater the stretching and contraction rates - precise definitions are given in $\S 5$ - in the chaotic bubble region. These two factors account for vastly different scalar distributions at different $A_{122}$. To what extent these advection-diffusion phenomena at small scalar diffusivity can be understood in terms of the dynamics under (2.8) is the content of the following sections.

\section{The zero-diffusivity solution and its relation to the solution at small scalar diffusivity}

Given some initial distribution of a scalar field one can construct a zero-diffusivity solution, also called the frozen-field solution, at any $t>0$ by treating $\theta$ as a material invariant. It might be expected that the effect of small scalar diffusion is to smooth out any fine-scale structure in the zero-diffusivity solution. Recent work of Klapper (1992a) has contributed strongly towards this description. If small scalar diffusion does no more than smooth out the fine variations in the zero-diffusivity solution, the distribution of the scalar field at small scalar diffusivity should retain the gross features of the zero-diffusivity solution. However, our problem is complicated by the fact that the zero-diffusivity solution, i.e. the solution of (3.1) at $P e=\infty$, is trivially zero over the entire domain of the flow. We therefore interpret the zero-diffusivity solution in the following sense: for $\mathrm{Pe} \rightarrow \infty, \mathrm{Pe}$ however large, there is always a thermal boundary layer near the active portion of the wall which is diffusively dominant and where diffusion may not be ignored. For $t=t_{0}$ sufficiently small, a solution in the boundary layer is easily obtained, which is used as an 'initial profile', $\theta\left(x, t=t_{0}\right)$, to obtain a 'zero-diffusivity' solution in the rest of the domain at $t>t_{0}$ by setting the scalar diffusivity to be exactly zero and treating $\theta$ as a material invariant for all $t>t_{0}$. The construction is artificial, but it proves to be a useful artifice in understanding not only the role of the unstable manifold as a organizing structure but also the role of small scalar diffusion as a local smoothing of fine-scale structure in the frozen field.

In the boundary layer at the wall, for $t$ sufficiently small the normal coordinate $x_{2}<O(1)$ for all $x_{1}$. If the lateral coordinate $x_{1}$ is $O(1)$, the advective term in (3.1), $\boldsymbol{u} \cdot \nabla \theta$, is $O\left(x_{2}\right)$, and therefore there is a time $t$ sufficiently small such that the advective term cannot match $\partial \theta / \partial t$ and will not contribute to dominant order. Moreover, for $x_{2} \ll x_{1}$, diffusion in the normal direction dominates over diffusion parallel to the wall. Hence, at small $t$ the dominant balance argument is straightforward: the transient term $\partial \theta / \partial t$ must be balanced by diffusion normal to the wall. In physical terms, for small $t$, in particular $t$ so small that advection is negligible in comparison to 
transverse diffusion over a narrow boundary layer at the wall, the advection-diffusion equation in the boundary layer must reduce to a simple transient diffusion equation with solution $\theta\left(x_{1}, x_{2}, t\right)$ independent of the lateral coordinate $x_{1}$. We rescale time, $t \rightarrow P e^{-\alpha} t, \alpha>0$. Balancing appropriate terms in (3.1) gives $x_{2}=O\left(P e^{-(\alpha+1) / 2}\right)$. Rescaling the normal coordinate in the near-wall boundary layer, $x_{2} \rightarrow P e^{-(\alpha+1) / 2} x_{2}$ gives a simple transient diffusion equation, to dominant order:

$$
\frac{\partial \theta}{\partial t}=\frac{\partial^{2} \theta}{\partial x_{2}^{2}},
$$

with initial and boundary conditions

$$
\begin{array}{r}
\theta(t \rightarrow 0)=0, \\
\theta\left(x_{2} \rightarrow 0\right)=1, \\
\theta\left(x_{2} \rightarrow \infty\right)=0,
\end{array}
$$

which has the familiar error function solution, expressed now in the unscaled coordinates,

$$
\theta\left(x_{1}, x_{2}, t\right)=1-\frac{2}{\pi^{1 / 2}} \int_{0}^{\frac{P_{e}^{1 / 2} x_{2}}{2 t^{1 / 2}}} \mathrm{e}^{-s^{2}} \mathrm{~d} s .
$$

The exact scaling factor $\alpha$ remains undetermined. The dominant balance argument is not expected to hold near the separation point $p^{-}$where advection is not dominantly parallel to the wall. But, for large $P e$, this advection-diffusion regime will be of negligible size and may be ignored. Comparison with the exact Wiener bundle solution for $\beta=0.6, \omega=0.72$, and $P e=2.5 \times 10^{4}$ along the centreline $\left(x_{1}=0, x_{2}>0\right)$ at several $t$ (see figure 16) shows that the error function solution of (4.1) tracks the time-evolution of the scalar field in the boundary layer all the way up to about $t \approx 10$, but deteriorates rapidly at larger times.

Using the error function solution of (4.1) for $P e=2.5 \times 10^{4}$ and $t=t_{0}$ sufficiently small as the 'initial distribution' $\theta\left(x, t=t_{0}\right)$, the 'zero-diffusivity' solution is obtained at several $t>t_{0}$, excluding of course the thin near-wall boundary-layer region (see figures 17,18 ). Henceforth, it will be understood that by 'zero-diffusivity' solution we mean $\theta\left(x, t>t_{0}\right)$ obtained using the 'initial distribution' described above. It is evident from figures 17 and 18 that the initial distribution, which is confined to the near-wall region, is wrapped around $W_{\beta}^{u}(\bar{\phi})$ as time progresses; as usual the numerical results are obtained at $t=N T$ for several $N$, i.e. at times that are integral multiples of the period $T$ of the velocity field, so that $\bar{\phi}=0$ and comparisons can be made with $W_{\beta}^{u}$ of figure 2. This result can be understood in simple terms by examining the dynamics of points in the near-wall region under forward iterations of the Poincare map $P_{\beta}$. Consider the heteroclinic tangle formed by the intersections of $W_{\beta}^{s}$ and $W_{\beta}^{u}$. If they intersect once, they must intersect a countably infinite number of times since their intersection points, called heteroclinic points (see Wiggins 1992), asymptote to the attachment point $p^{+}$in forward time and to the separation point $p^{-}$in backward time, and therefore constitute a doubly asymptotic set. Moreover, if they intersect transversely, there exists a countable infinity of transverse heteroclinic points. This is due to the fact that transversal intersections are preserved under diffeomorphisms and $P_{\beta}$, being the time- $T$ map derived from a smooth flow, is a diffeomorphism. Hence the presence of the plane wall and the accumulation of transverse heteroclinic points close to $p^{-}$, in terms of arclength along $W_{\beta}^{u}$, forces segments of $W_{\beta}^{s}$ to accumulate at 


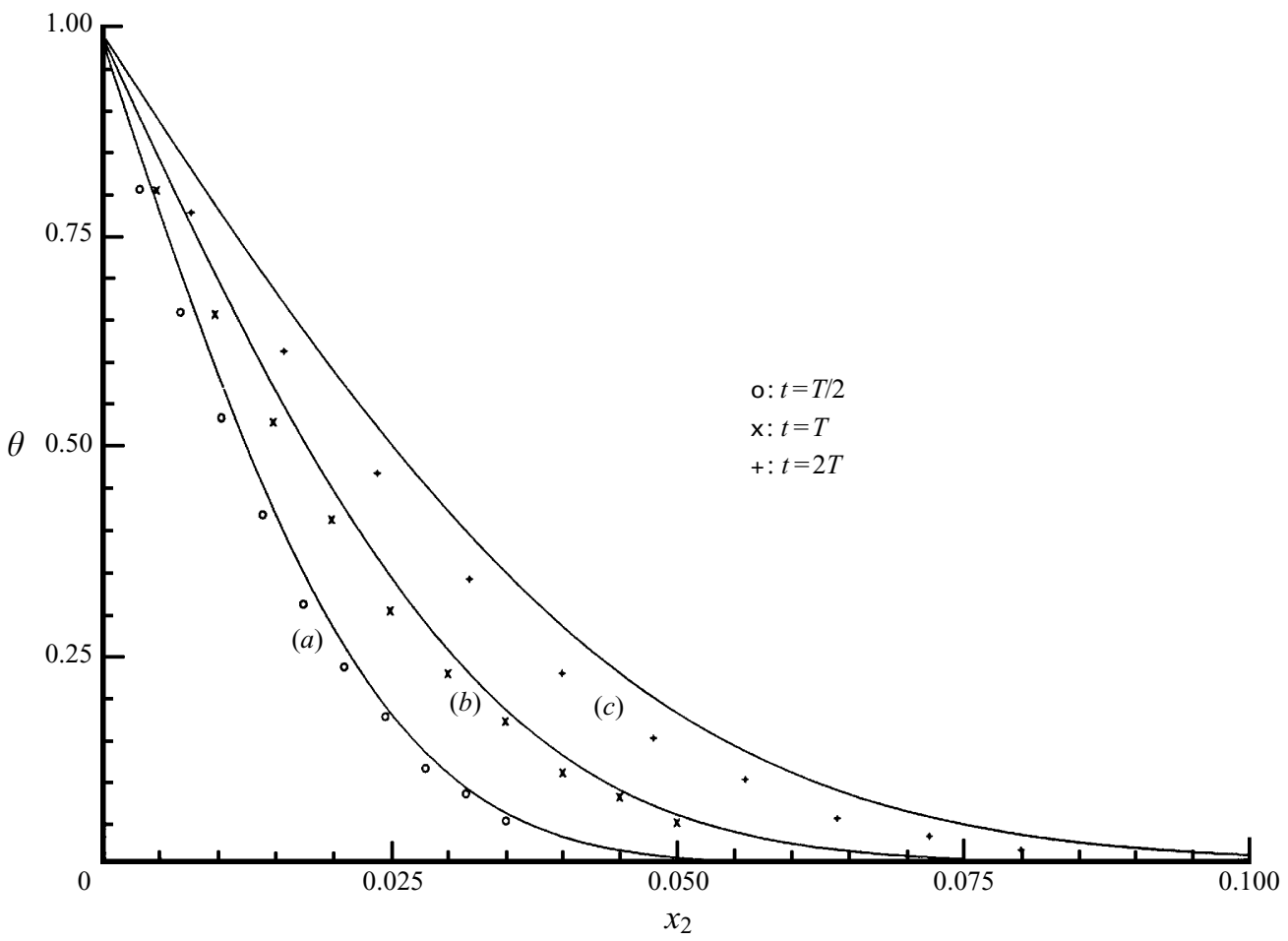

FIGURE 16. Wiener bundle solutions (symbols) at several points along the centreline $\left(x_{1}=0, x_{2}>0\right)$ at several times and for $\beta=0.6, \omega=0.72, P e=2.5 \times 10^{4}$, are compared to the small-time analytical solution of (4.1) at the same Pe (solid lines). (a) $t=T / 2$, (b) $t=T,(c) t=2 T, T=2 \pi / \omega$.

the wall, giving rise to the familiar trellis-type structure studied originally by Poincare (e.g. see Easton 1986). This is demonstrated by the heteroclinic tangle over the wall, obtained in figure 19 for $\beta=0.6, \omega=0.72$.

Integration errors near the no-slip boundary makes resolution of the tangle difficult, but the afore-mentioned trellis-type structure of the tangle is clearly evident. Now consider the countable infinity of transverse heteroclinic points $p_{i}, i \in Z$, that accumulate on $p^{-}$in the sense described above. As segments of $W_{\beta}^{s}$ densely fill out the near-wall region $R_{w} \equiv\left(-1.5,1-\epsilon_{1}\right) \times\left(0, \epsilon_{2}\right), 0<\epsilon_{1}, \epsilon_{2} \ll 1, \epsilon_{1} \rightarrow 0$ as $\epsilon_{2} \rightarrow 0$, every open neighbourhood of any point in $R_{w}$ has points lying on a segment of $W_{\beta}^{s}$ or on the stable manifold through some $p_{i}$, where the stable manifold through $p_{i}$ is the set of points that converge asymptotically to the trajectory of $p_{i}$ under forward iterations of $P_{\beta}$. Since points on $W_{\beta}^{s}$ converge asymptotically in forward time, and the $p_{i}$ are mapped to transverse heteroclinic points under $P_{\beta}$, points in $R_{w}$ are swept into a thin neighbourhood of $W_{\beta}^{u}$ under forward iterations of $P_{\beta}$, which accounts for the nature of the zero-diffusivity solution observed in figures 17, 18. Moreover, because of the asymptotic convergence of points on $W_{\beta}^{s}$, the zero-diffusivity solution will have a steep gradient in the local stable direction which, for a transverse heteroclinic point, is the direction of the tangent to $W_{\beta}^{s}$ at that point and is complementary to the tangent direction of $W_{\beta}^{u}$ at that point. This is verified by extracting from figure 17 the scalar distribution in directions tangent and normal to $W_{\beta}^{u}$ at a point $p \in W_{\beta}^{u}$, i.e. along $T_{p}\left(W_{\beta}^{u}\right)$ and $N_{p}\left(W_{\beta}^{u}\right)$, which are displayed in figure 20.

We make a slight digression to address a technical point. While figure 19 does supply 

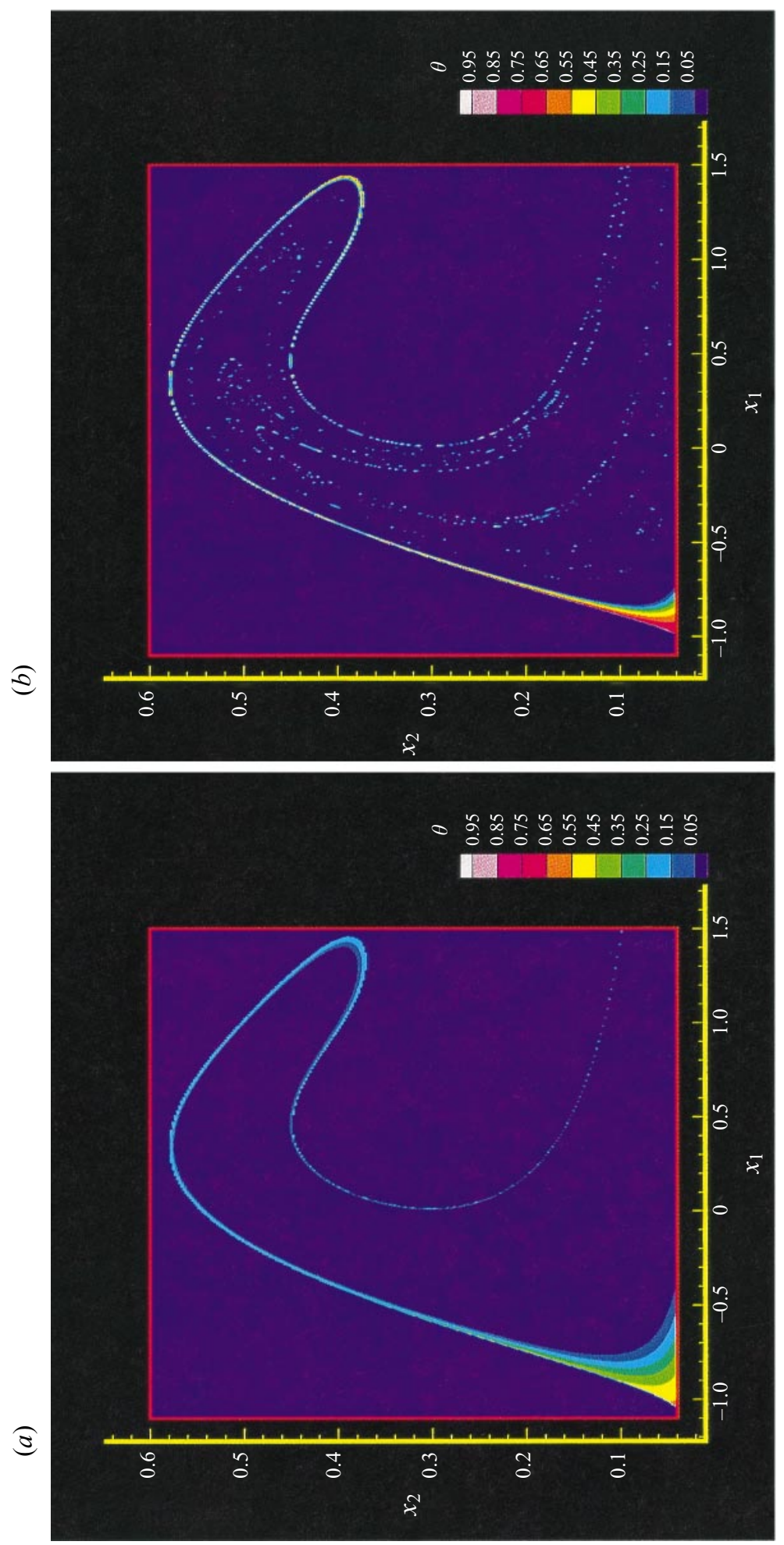

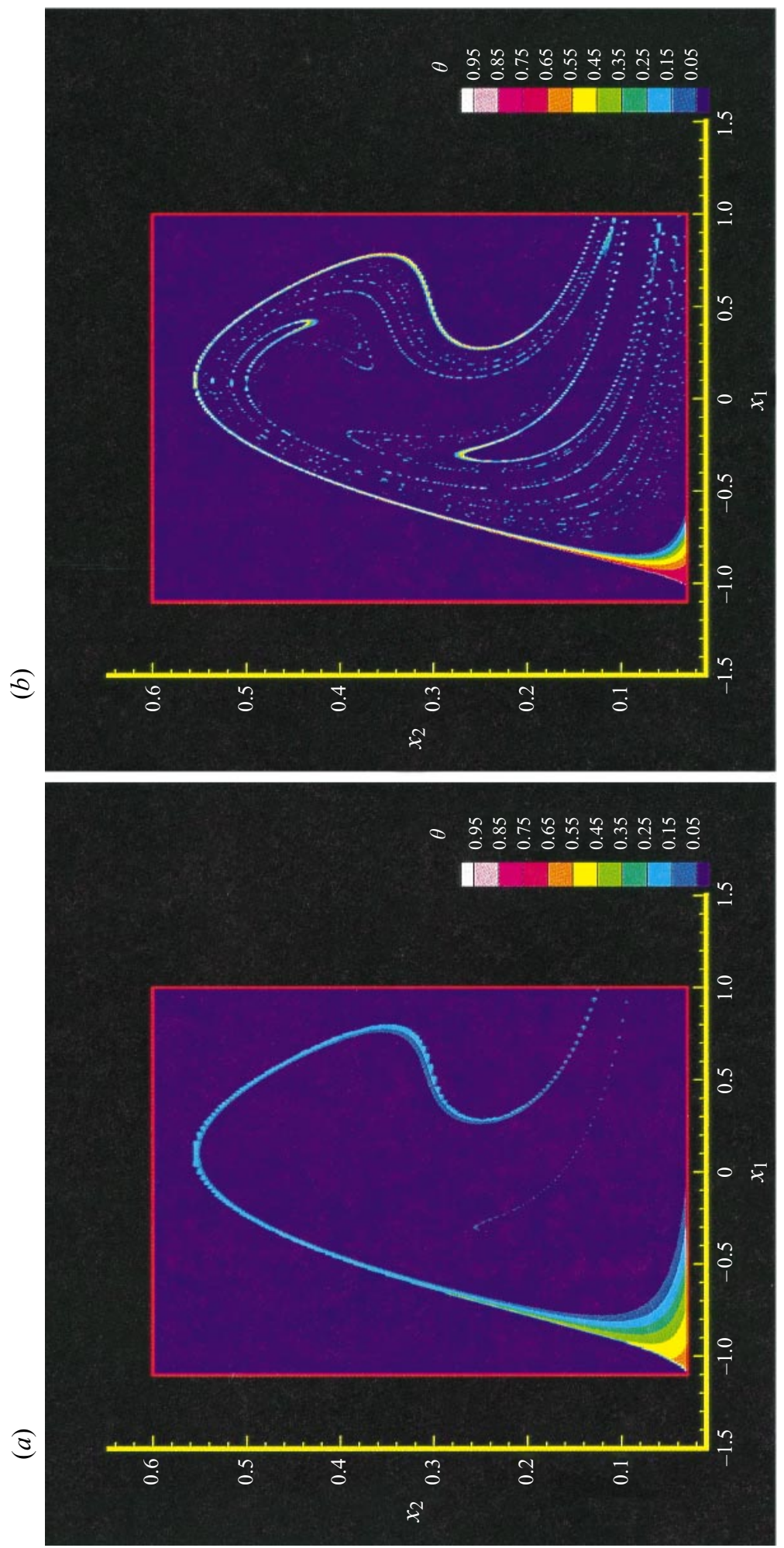

. 


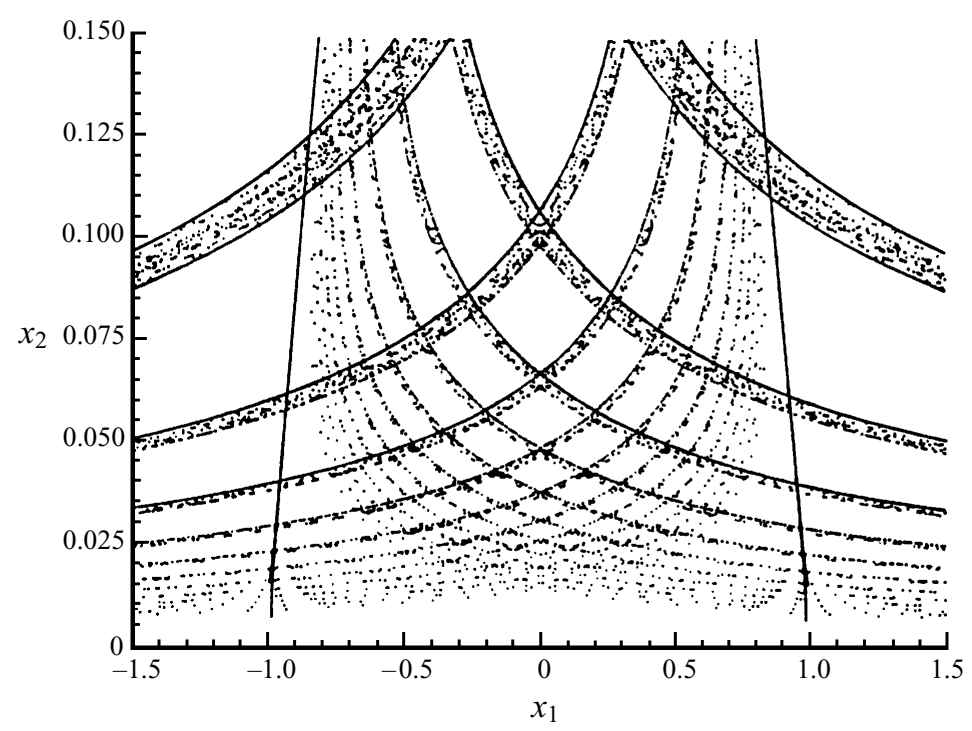

FIGURE 19. Heteroclinic tangle near the wall for $\beta=0.6, \omega=0.72$.
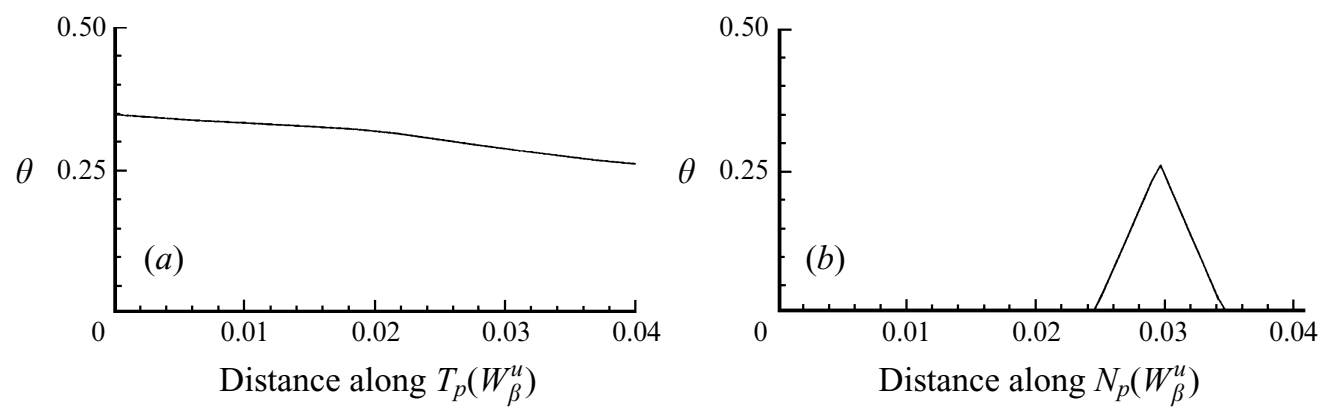

FIGURE 20. Distribution of the scalar field at $t=10 T$ in the zero-diffusivity solution of figure 17 along $(a) T_{p}\left(W_{\beta}^{u}\right),(b) N_{p}\left(W_{\beta}^{u}\right), p=(-0.547,0.321) \in W_{\beta}^{u}$. The point $p$ is plotted in figure $2(a)$.

numerical evidence of transversal intersections of $W_{\beta}^{s}$ and $W_{\beta}^{u}$, analytical verification can also be obtained using the Melnikov function (Wiggins 1992) which gives a signed measure of the distance between $W_{\beta}^{s}(\bar{\phi})$ and $W_{\beta}^{u}(\bar{\phi})$. Assuming smoothness of $W_{\beta}^{s}(\bar{\phi})$ and $W_{\beta}^{u}(\bar{\phi})$, Melnikov's theory can be used to obtain the following Melnikov function for our problem, including complications due to the non-hyperbolicity of $p^{-}$and $p^{+}$ (e.g. see Camassa \& Wiggins 1991 and Ghosh 1994):

$$
M(\tau)=A(\omega) \sin (\omega \tau)
$$

where $\tau$ parametrizes the heteroclinic connection $\psi_{h}$, and

$$
A(\omega)=\int_{-\infty}^{+\infty}\left(f_{1}^{u} g_{2}^{u}-f_{2}^{u} g_{1}^{u}\right)\left(x_{h}(t)\right) \cos (\omega t) \mathrm{d} t
$$

where $f^{u} \equiv\left(f_{1}^{u}, f_{2}^{u}\right)$ and $g^{u} \equiv\left(g_{1}^{u}, g_{2}^{u}\right)$ are from the velocity field of (2.8), and $x_{h}(t)$ is the heteroclinic orbit. For $A(\omega) \neq 0$, it is a straightforward conclusion that $W_{\beta}^{s}$ and $W_{\beta}^{u}$ do intersect transversely (Wiggins 1990). The variation of $A(\omega)$ with $\omega$ is shown in figure 21 . We also note that the perturbation frequency $\omega$ was chosen to be 0.72 


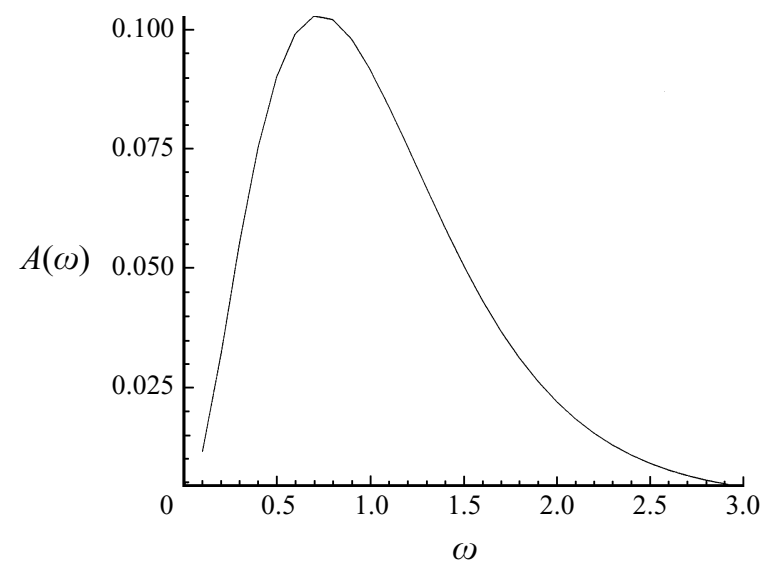

FIGURE 21. Variation of amplitude $A$ of the Melnikov function with perturbation frequency $\omega$.

in our computations since $A(\omega)$ is maximum at $\omega=0.72$; it is desirable to have large $A(\omega)$ since the width of the chaotic layer increases with $A(\omega)$ (Ghosh, Chang \& Sen 1992). Moreover, it is evident from figure 21 that the distance between the stable and unstable manifolds falls rapidly as $\omega$ is increased beyond 0.72 and for such $\omega, W_{\beta}^{s}$ and $W_{\beta}^{u}$ do not sweep out the entire bubble region; this is certainly true for $\omega=2.0$.

Thus the zero-diffusivity solution at $t>t_{0}$ pulls the boundary layer distribution at $t=t_{0}$ into a narrow distribution along the unstable manifold of $p^{-}, W_{\beta}^{u}(\bar{\phi})$, where the variation of the scalar field along $W_{\beta}^{u}(\bar{\phi})$ is much smaller than in transverse directions. The physical picture arising from comparing figures $17(a, b), 18(a, b)$ with figures $3(a, b), 4(a, b)$ respectively is that the zero-diffusivity solution creates fine-scale structure that is confined to the vicinity of $W_{\beta}^{u}(\bar{\phi})$ and small scalar diffusion only smooths out the fine variations, thus allowing the unstable manifold to continue to be the dominant organizing structure everywhere except in a narrow diffusively-dominant near-wall boundary layer. In the following section we develop an understanding of this qualitative picture using recent results (Klapper 1992a) based on the shadowing theory of chaotic hyperbolic sets.

\section{Application of shadowing theory to the small diffusivity problem}

Klapper (1992a) has shown how shadowing theory from deterministic dynamical systems theory can be used to study the small diffusivity problem. We apply his approach to our problem, where it enables us to understand:

(1) the role of diffusion as a smoothing of the zero-diffusivity solution at small diffusivity,

(2) the approach of the scalar field to an asymptotic state over an intermediate time scale,

(3) the observed increase in non-uniformity of the scalar field as the perturbation is increased,

(4) the role of the unstable manifold of $p^{-}$as the geometric structure in the flow governing the spatial distribution of the scalar field.

We now describe the method. We consider a discrete-time formulation in which instantaneous advection, given by the Poincare map $P_{\beta}^{\bar{\phi}}$, is alternated with pulses of diffusion over time $T$. Therefore, a diffusive tracer located at $x^{0}$ at initial time $t_{0}$ is 
advanced according to

$$
x^{n+1}=f\left(x^{n}\right)+\rho_{T}, n=0,1, \ldots,
$$

with $\rho_{T} \equiv\left(\eta_{T}, \xi_{T}\right)$, where $\eta_{T}, \xi_{T}$ are Gaussianly distributed random variables, each with mean zero and variance $2 T / P e$, diffeomorphism $f \equiv P_{\beta}^{\bar{\phi}}$, and the cross-section $\bar{\phi}$ is determined by $\bar{\phi}=\omega t_{0}(\bmod 2 \pi)$.

The shadowing lemma applies to the deterministic part of (5.1), i.e. the map $f$. Strictly speaking, it only applies in a region where every point has a one-dimensional exponentially attracting direction and a one-dimensional exponentially expanding direction, and the angle between these two directions is bounded away from zero. Regions having this property are called hyperbolic sets, and we will denote such a region by $\Lambda$. The shadowing lemma (Bowen 1975) states that for each $\delta>0$ there is an $\epsilon>0$, such that every trajectory $\left\{x^{i}\right\}_{i=n}^{m}$ in $\Lambda$ satisfying $\left|x^{i+1}-f\left(x^{i}\right)\right|<\epsilon$ for all $n \leqslant i \leqslant m$ (called an $\epsilon$-pseudo-orbit) is $\delta$-traced by an exact trajectory $\left\{y^{i}\right\}_{i=n}^{m}$ in $\Lambda$ (called the shadowing trajectory), i.e. $y^{i+1}=f\left(y^{i}\right)$ and $\left|x^{i}-y^{i}\right| \leqslant \delta$ for all $n \leqslant i \leqslant m$, where $n=-\infty$ and $m=+\infty$ is permitted. For $\Lambda \subset \mathbb{R}^{2},|\cdot|$ can be taken to be the standard Euclidean norm on the plane. If the shadowing lemma can be applied (a question that we will address more completely after we describe the method), the picture we have in mind is that the $\epsilon$-pseudo-orbits represent diffusive or Wiener trajectories. Then the shadowing lemma implies that such trajectories are approximated by deterministic trajectories.

In what follows, we assume that the dynamics at every point can indeed be decomposed into a stable and unstable direction. Consider then a diffusive tracer located at $x^{N}$ and travelling backwards under (5.1), i.e.

$$
x^{n-1}=f^{-1}\left(x^{n}\right)+\rho_{T}, \quad n \in[1, N] .
$$

Therefore (5.2) generates a bundle or cloud of Wiener trajectories, each of which starts at a random 'Wiener initial point' $x^{0}$ at iteration zero or $t=t_{0}$ and arrives at the fixed non-random point $x^{N}$ in $N$ forward iterations. Now, every Wiener trajectory generated by (5.2) has a noisy deviate $\rho_{T}$ at each iterate which is typically $O\left((T / P e)^{1 / 2}\right)$. Because of the Gaussian tail, $\rho_{T}$ can become unbounded in some realizations, but the probability of such an event can be made arbitrarily small for any finite number of realizations by taking $P e$ large enough and thus forcing the variance of the noisy deviates to be as small as desired. More precisely, given an $\epsilon>0$, choosing $P e$ sufficiently large ensures that the sample paths of (5.2), with probability arbitrarily close to unity, have noisy deviates that are bounded from above by $\epsilon$ at every time-step over finite time-intervals. Therefore, at large Pe almost all Wiener trajectories $\left\{x^{n}\right\}_{n=N}^{0}$ generated by (5.2) are $\epsilon$-pseudo-orbits, and these will be shadowed as long as the trajectory is confined to regions of the flow where the shadowing property holds. Notice the difficulty in extending the argument to infinite times: as $N \rightarrow \infty$, every Wiener trajectory will, with probability one, experience a large noise event even at arbitrarily large $P e$ and cannot be shadowed. However, it is irrelevant to our problem since we are interested in only finite-time results. Recall from (3.5) that computing the Wiener bundle solution for the scalar value at the point $x^{N}$ at time $t$ requires taking an expectation over this bundle of Wiener trajectories. Since the exact distribution of Wiener trajectories is difficult to obtain in an arbitrary flow, the method might seem to offer little additional insight. However, chaotic flows with the shadowing property and the associated hyperbolic structure 
provide a means of representing the distribution of Wiener trajectories in terms of exact shadow trajectories in a convenient way (Klapper $1992 a$ ).

Consider the Wiener trajectory $\left\{x^{n}\right\}_{n=N}^{0}$ generated using (5.2). The first noisy backward iterate of $x^{N}$ is $O\left((T / P e)^{1 / 2}\right)$ close to $f^{-1}\left(x^{N}\right)$. In the next backward iterate, the component of $x^{N-1}$ in the local unstable direction of $f^{-1}\left(x^{N}\right)$ is contracted exponentially under $f^{-1}$ and therefore $x^{N-2}$ remains $O\left((T / P e)^{1 / 2}\right)$ close to the stable manifold of $f^{-2}\left(x^{N}\right)$. Continuing in this way, the $N$ th noisy backward iterate of $x^{N}, x^{0}$ is $O\left((T / P e)^{1 / 2}\right)$ close to the stable manifold through $f^{-N}\left(x^{N}\right)$, which is also the $N$ th preimage of the stable manifold through $x^{N}$. Denoting the stable manifold through $x^{N}$ as $W^{s}\left(x^{N}\right)$, the hyperbolic structure of the dynamics pulls the cloud of Wiener trajectories released at $x^{N}$ into an $O\left((T / P e)^{1 / 2}\right)$ neighbourhood (which can be imagined as a tube or sausage of thickness $\left.O\left((T / P e)^{1 / 2}\right)\right)$ of $f^{-N}\left(W^{s}\left(x^{N}\right)\right)$ in $N$ backward time-steps. The observation is crucial and was first made by A. D. Gilbert (see Klapper $1992 b$ ). From (3.5), the Wiener bundle solution at $x^{N}$ at time $t=N T+t_{0}$ is therefore an appropriately weighted average of the initial field distribution $\theta\left(x, t_{0}\right)$ in an $O\left((T / P e)^{1 / 2}\right)$ neighbourhood of the $N$ th preimage of the stable manifold through $x^{N}$. If $\theta\left(x, t_{0}\right)$ is continuous, assuming all Wiener initial points, i.e. the $x^{0}$, lie on $f^{-N}\left(W^{s}\left(x^{N}\right)\right)$ causes an error of $O\left((T / P e)^{1 / 2}\right)$ in $\theta\left(x^{N}, t=N T+t_{0}\right)$, and can be ignored. Thus the assumed hyperbolic structure of the dynamics leads to an important dimensional reduction in the problem, since obtaining the Wiener bundle average of (3.5) now requires computing the distribution of Wiener initial points in terms of arclength along the one-dimensional manifold $f^{-N}\left(W^{s}\left(x^{N}\right)\right)$. Note, however, that the variance of this distribution can be $O(1)$; due to the fast (asymptotically exponential) convergence of points on the stable manifold in forward time, the noisy backward iterates $x^{N-n}$ can quickly move an $O(1)$ distance away from $f^{-n}\left(x^{N}\right)$ in terms of arclength along $f^{-n}\left(W^{s}\left(x^{N}\right)\right)$, even for very large $P e$. Therefore, the averaging might have to be carried out over a $O(1)$ length of $f^{-N}\left(W^{s}\left(x^{N}\right)\right)$. But, since $f^{-N}\left(W^{s}\left(x^{N}\right)\right)$ is not an embedding in $\mathbb{R}^{2}$ it is not a submanifold of $\mathbb{R}^{2}$ and can be a very complicated geometric object, in which case no major simplification is achieved. The shadowing property offers a way around this problem. From the shadowing lemma and our discussion above, almost every Wiener trajectory is $\delta$-shadowed by an exact trajectory and $\delta$ can be chosen arbitrarily small provided $P e$ is sufficiently large. A Wiener trajectory $\left\{x^{n}\right\}_{n=N}^{0}$ and the corresponding exact shadow trajectory $\left\{y^{n}\right\}_{n=N}^{0}$ will pick up the same scalar value to $O(\delta)$ and therefore the contribution to the Wiener bundle average of $(3.5)$ by $\theta\left(x^{0}, t_{0}\right)$ can be replaced by $\theta\left(y^{0}, t_{0}\right)$ with asymptotically small error. It should be clear that the $y^{0}$ must lie on $f^{-N}\left(W^{s}\left(x^{N}\right)\right)$; otherwise, since the component in the unstable direction is exponentially expanded in forward time, forward iterates of $y^{0}$ (under the deterministic map, $f$ ) will move quickly away from the preimages of the stable manifold through $x^{N}$ and cannot remain close to $\left\{x^{n}\right\}_{n=N}^{0}$. The advantage gained is the following: the distribution of Wiener initial points over $f^{-N}\left(W^{s}\left(x^{N}\right)\right)$ can be mapped back to $W^{s}\left(x^{N}\right)$ under $f^{N}$, since the Wiener trajectories can be replaced by exact trajectories of $f^{N}$ to $O(\delta)$, yielding the appropriate weighting law for averaging the zero-diffusivity solution over $W^{s}\left(x^{N}\right)$. Since points on $f^{-N}\left(W^{s}\left(x^{N}\right)\right)$ converge exponentially under $f^{N}$ as $N \rightarrow \infty$, the variance of the distribution over $f^{-N}\left(W^{s}\left(x^{N}\right)\right)$ is also asymptotically exponentially contracted under $f^{N}$, which is an important simplification since $W^{S}\left(x^{N}\right)$ can be replaced by a straight line $E^{s}\left(x^{N}\right)$ in the local stable direction at $x^{N}$. Therefore, the Wiener bundle average of the initial field $\theta\left(x, t_{0}\right)$ over an $O\left((T / P e)^{1 / 2}\right)$ neighbourhood of $f^{-N}\left(W^{s}\left(x^{N}\right)\right)$ is asymptotically replaced by a properly weighted average of 
the zero-diffusivity solution over $E^{s}\left(x^{N}\right)$ in the Wiener bundle solution of (3.5) at $x^{N}$ at $t=N T+t_{0}$. Klapper (1992a) showed that the asymptotic $(P e \rightarrow \infty)$ weighting law for averaging the zero-diffusivity solution over $E^{s}\left(x^{N}\right)$ is Gaussian, with zero mean and variance

$$
\sigma_{N}^{2}=\frac{2 T}{P e} \sum_{n=1}^{N}\left(\frac{1}{\lambda_{n}}\right)^{2},
$$

where $\lambda_{n}>1$ is the expansion factor of $f^{-n}$ along the local stable direction at $x^{N}$. The $\lambda_{n}$ are easily expressed in terms of the local expansion rates at every iterate. Let $f_{n}$ be the restriction of $f^{-1}$ to $W^{s}\left(f^{-n+1}\left(x^{N}\right)\right)$ and introduce $F_{n}=f_{n} \circ f_{n-1} \circ \cdots \circ f_{1}$; $F_{n}$ maps $W^{s}\left(x^{N}\right)$ onto $W^{s}\left(f^{-n}\left(x^{N}\right)\right)$. Then $\lambda_{n}$ is simply

$$
\begin{aligned}
\left.\lambda_{n} \equiv \mathrm{d} F_{n}\right|_{x^{N}} & =\left.\left.\left.\mathrm{d} f_{n}\right|_{f^{-n+1}\left(x^{N}\right)} \circ \mathrm{d} f_{n-1}\right|_{f^{-(n-1)+1}\left(x^{N}\right)} \circ \cdots \circ \mathrm{d} f_{1}\right|_{x^{N}}, \\
& =\beta_{n} \circ \beta_{n-1} \circ \cdots \circ \beta_{1},
\end{aligned}
$$

where $\beta_{i}=\left.\mathrm{d} f_{i}\right|_{f^{-i+1}\left(x^{N}\right)}$ is the expansion factor of $f^{-1}$ in the local stable direction of $f^{-i+1}\left(x^{N}\right)$.

Finally, we discuss conditions under which the shadowing lemma applies. The result would appear to be useful only in the special case of uniformly hyperbolic or everywhere hyperbolic (Farmer \& Sidorowich 1991) dynamical systems (also called Anosov dynamical systems) in which at each point the dynamics can be decomposed into local stable and unstable directions and the stable and unstable manifolds always intersect transversely, i.e. there are no homoclinic tangencies (Farmer \& Sidorowich 1991). However, work of Klapper (1992a) has indicated that making the assumption that the shadowing property holds in non-uniformly hyperbolic systems such as ours can lead to useful insights into the small-diffusivity scalar advection-diffusion problem. Our work also confirms this hope, and we support this with numerical simulations that enable us to determine when shadowing breaks down (i.e. when homoclinic tangencies occur). A detailed numerical study of this for our problem can be found in the thesis of Ghosh (1994).

\subsection{Comparison of shadowing and the Wiener bundle methods}

In figure $22(a) 10^{4}$ Wiener trajectories starting at $p_{1}=(0.25,0.455)$ are generated using (5.2) by advancing backward in time up to a length of time $5 T$, for $\beta=0.6, \omega=0.72$, and $P e=10^{6}$, to obtain the distribution of Wiener initial points in the domain of interest, which is the bubble region or the flow region above the active portion of the wall. An equal number of points is distributed uniformly along the stable direction at $p_{1}$ up to a length $3 \sigma$ on either side of $p_{1}$, where $\sigma$ is the standard deviation of the Gaussianly distributed shadowing trajectories, computed for the same parameter values as above, using (5.3). These are integrated backwards up to a length of time $5 T$ using the velocity field of (2.8) to obtain the preimage of the stable manifold through $p_{1}$, and is shown in figure 22(b). It is evident that most of the mass of the distribution of Wiener initial points is confined to a small neighbourhood of the preimage of the stable manifold through $p_{1}$. These results illustrate the usefulness of the shadowing property.

5.2. Smoothing of the zero-diffusivity solution by diffusion at small scalar diffusivity Due to the hyperbolic character of the dynamics in chaotic flows, the fine-scale direction (or the scalar gradient) at a point in the zero-diffusivity solution will be, 

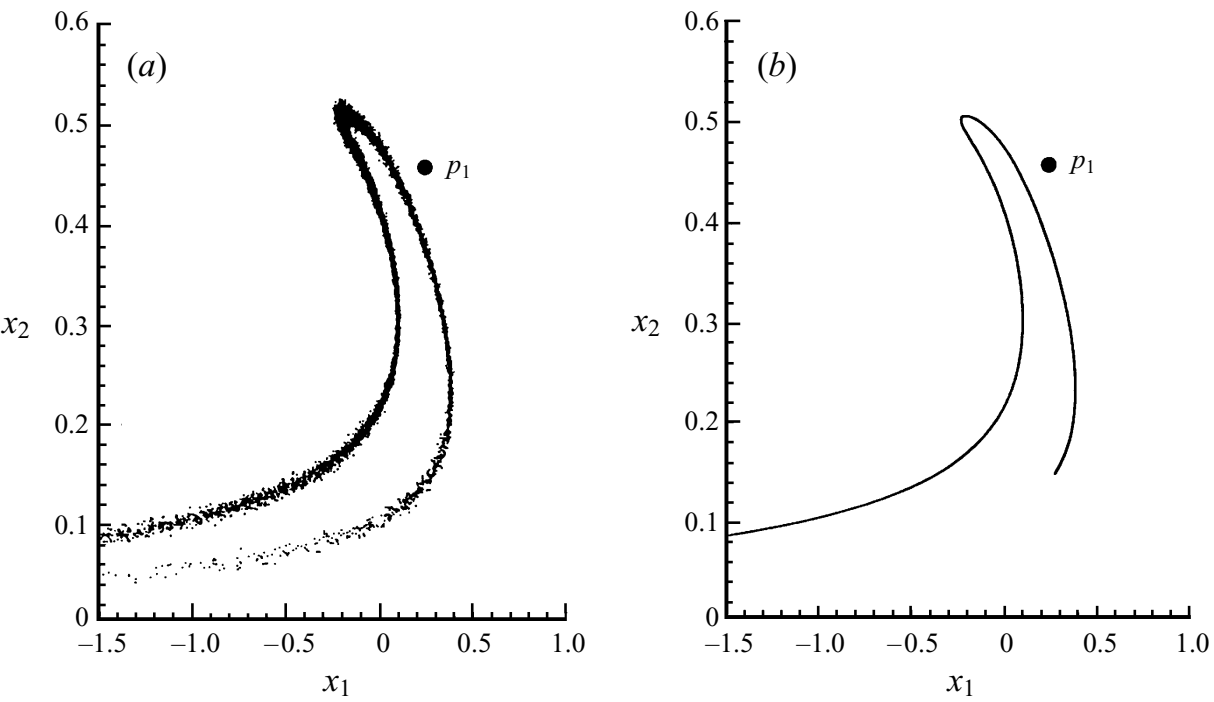

FIGURE 22. (a) Distribution of $10^{4}$ Wiener initial points that arrive at the point $p_{1}=(0.25,0.455)$ in time $t=5 T$. Relevant parameter values are $\beta=0.6, \omega=0.72, P e=10^{6}$, and $T=2 \pi / \omega$. $(b) 10^{4}$ points lined up along the stable direction at $p_{1}=(0.25,0.455)$ up to a length $3 \sigma$ on either side of $p_{1}$ and integrated backwards for a length of time 5T, where $\sigma=0.0015$ and is computed using (5.3) for parameter values identical to $(a)$.

roughly speaking, in the local stable direction (Klapper 1992a). For a point $p$ on the unstable manifold of $p^{-}, p \in W_{\beta}^{u}(\bar{\phi})$, the local unstable direction is $T_{p}\left(W_{\beta}^{u}(\bar{\phi})\right)$, while the local stable direction is in general complementary to $T_{p}\left(W_{\beta}^{u}(\bar{\phi})\right)$, unless $p$ is a point of homoclinic tangency; not surprisingly, scalar profiles extracted along $T_{p}\left(W_{\beta}^{u}\right)$ and in the direction orthogonal to $T_{p}\left(W_{\beta}^{u}\right)$ are strikingly different (see figure 20). Therefore, as long as the shadowing property holds, adding small scalar diffusion will smooth fine-scale structure in the zero-diffusivity solution around $p \in W_{\beta}^{u}(\bar{\phi})$. Since the zero-diffusivity distribution is almost entirely confined along $W_{\beta}^{u}(\bar{\phi})$, adding small scalar diffusion only smears out the zero-diffusivity solution about $W_{\beta}^{u}(\bar{\phi})$.

\subsection{Further considerations of the zero-diffusivity solution}

Before we can apply shadowing theory to determine the effects of small diffusivity, we need to continue our analysis and characterization of the zero-diffusivity solution begun in $\S 4$. From both theoretical arguments and numerical evidence presented in $\S 4$, it is clear that the zero-diffusivity solution away from the wall is confined entirely to the vicinity of the unstable manifold of $p^{-}, W_{\beta}^{u}$, and therefore it will be sufficient to consider the dynamics of points in the vicinity of $W_{\beta}^{u}$ even for the full advection-diffusion problem. Let $\bar{R}_{w}=(-1.5,1) \times\left(0, \epsilon_{1}\right)$, where $\epsilon_{1}=O\left(P e^{-(\alpha+1) / 2}\right)$. Therefore $\bar{R}_{w}$ is the thin near-wall region in which the 'initial' distribution $\theta\left(x, t_{0}\right)$ is non-zero. Let $\theta_{f r}$ denote the zero-diffusivity or frozen-field solution. Consider a point $x$ in the vicinity of $W_{\beta}^{u}$, and let $\left\{x^{n}\right\}_{n=N}^{0}$ be the exact dynamical trajectory through $x$ under backward iterations of $f$, i.e. $x^{N} \equiv x$. Then, $\theta_{f r}\left(x, t=N T+t_{0}\right) \neq 0$ if and only if $f^{-N}\left(x^{N}\right) \equiv x^{0} \in \bar{R}_{w}$. Let $x \in W^{s}(y)$, where $y \in W_{\beta}^{u}$, and let $\left\{y^{n}\right\}_{n=N}^{0}$ be the exact dynamical trajectory through $y$ under backward iterates of $f, y^{N} \equiv y$. Moreover, let $N_{1}$ be defined such that for $N>N_{1}\left(\epsilon_{1}, y\right),\left|f^{-N}\left(y^{N}\right)-p^{-}\right|<\epsilon_{1}$, where recall that $p^{-}$ 
is the point of separation on the no-slip boundary. Then, if $x^{0} \in \bar{R}_{w}$,

$$
\left|x^{0}-y^{0}\right| \simeq \lambda_{N}|x-y|,
$$

where the segment of $W^{s}\left(y^{0}\right)$ in $\bar{R}_{w}$ is being approximated as a straight line and $|x-y|$ is assumed small; $\lambda_{N}$ is the expansion factor of $f^{-N}$ along the local stable direction of $y \in W_{\beta}^{u}$. Therefore $\theta_{f r}\left(y, t=N T+t_{0}\right) \neq 0$ for all $N>N_{1}\left(\epsilon_{1}, y\right)$ and from (5.4), the width of the zero-diffusivity solution at $y$ will be $O\left(1 / \lambda_{N}\right)$, where by 'width' we mean the length over which the zero-diffusivity solution is non-zero in the local stable direction at $y$.

Since the exact dynamical trajectory through a point on the unstable manifold $y \in W_{\beta}^{u}$ under backward iterations of $f$ approaches the separation point $p^{-}$and, since $p^{-}$is a non-hyperbolic stagnation point, the local expansion rates or $\beta_{n}$ along the backward trajectory approach unity. In fact one can show that (Ghosh 1994) $\left(\beta_{N}-1\right) \leqslant \epsilon_{0}$ for all $N>N_{0}$ where $N_{0}$ depends on $\epsilon_{0}$ and $y$. A verification is provided by numerical computations presented below (see figure 23 ).

Now, choosing $\epsilon_{0}$ very small, $N_{0}>N_{1}\left(\epsilon_{1}, y\right)$. Since for all $N>N_{0}$

$$
\lambda_{N}=\left(\beta_{N} \circ \cdots \circ \beta_{N_{0}+1}\right) \circ \lambda_{N_{0}}
$$

it easily follows that

$$
\lambda_{N} \leqslant\left(1+\epsilon_{0}\right)^{\left(N-N_{0}\right)} \lambda_{N_{0}} .
$$

Hence for all finite $N>N_{0}$

$$
\lambda_{N_{0}} \leqslant \lambda_{N} \leqslant \lambda_{N_{0}}+O\left(\epsilon_{0}\right) .
$$

There is therefore an intermediate time-scale specified by $\left(N-N_{0}\right)<O\left(1 / \epsilon_{0}\right)$ over which $\lambda_{N}$ is infinitesimally close to $\lambda_{N_{0}}$ and the width of the zero-diffusivity solution at $y$ is $O\left(1 / \lambda_{N_{0}}\right)$. We note that though the right-hand side of (5.5) diverges for $N \rightarrow \infty$, this limit is irrelevant to our discussion since shadowing theory applies only on finite time-scales. The zero-diffusivity solution is therefore $O\left(1 / \lambda_{N_{1}}\right)$ wide at $y \in W_{\beta}^{u}$ when it first appears at $N=N_{1}$, but shrinks to an asymptotic width of $O\left(1 / \lambda_{N_{0}}\right)$ as $N$ increases beyond $N_{0}$; of course since $N_{0}>N_{1}, \lambda_{N_{0}}>\lambda_{N_{1}}$.

Owing to the non-hyperbolicity of $p^{-}$, backward iterates of $y$ approach $p^{-}$very slowly. Since backward iterates of $x \in W^{s}(y), O\left(1 / \lambda_{N_{0}}\right)$ close to $y$, lie on segments of $f^{-N}\left(W^{s}(y)\right)$ that are approximately parallel to the wall, their $x_{2}$-coordinate varies very slowly with $N$ for $N$ large. Again, this can be made precise by considering the local representation of $f^{-1}$ in the vicinity of any point on the no-slip boundary (Ghosh 1994). Therefore the backward iterates pick-up approximately the same scalar value from the 'initial' distribution in $\bar{R}_{w}$. It is therefore concluded that on an intermediate time-scale specified by $N_{0}<N<N_{0}+O\left(1 / \epsilon_{0}\right)$ the zero-diffusivity solution at $y$, and in an $O\left(1 / \lambda_{N_{0}}\right)$ vicinity of $y$ along the local stable direction at $y$, does not change appreciably and can be said to have attained an asymptotic frozen-field distribution.

\subsection{The small-diffusivity solution}

From the viewpoint being considered here, the scalar value at a point in the bubble region, in the presence of small scalar diffusion, depends on twin factors: firstly, the zero-diffusivity distribution in the local stable direction at that point built-up by the underlying chaotic advection discussed above and secondly, the local shape of the asymptotic weighting function imposed by the smoothing process of scalar diffusion. We consider now the latter.

It is clear that if the role of small scalar diffusion is a local smoothing of scalar 
gradient built-up in the zero-diffusivity solution, then the scalar value at a point in the presence of small scalar diffusion will be zero if the zero-diffusivity solution is also zero at that point and in a neighbourhood of that point; the size of this neighbourhood will of course depend on the variance of the smoothing function, and from (5.3) it is evident that for finite-times and large $P e$, the size will be asymptotically small.

In addition, for all $N>N_{0}$, and for all $x \in W^{s}(y)$ such that $x$ is $O\left(1 / \lambda_{N_{0}}\right)$ close to $y$, the variance of the Gaussian weighting function of (5.3) satisfies

$$
\sigma_{N}^{2} \leqslant \frac{2 T}{P e}\left[\sum_{n=1}^{N_{0}} \frac{1}{\lambda_{n}^{2}}+\left(\frac{N-N_{0}}{\lambda_{N_{0}}^{2}}\right)\right],
$$

where use has been made of the fact that $\lambda_{n}$ is a non-decreasing function of $n$. For most points in the bubble-region away from the wall $\lambda_{N_{0}}$ is large. Therefore again, for all finite $N>N_{0}$,

$$
\sigma_{N_{0}}^{2} \leqslant \sigma_{N}^{2} \leqslant \sigma_{N_{0}}^{2}+O\left(\frac{1}{\lambda_{N_{0}}^{2}}\right)
$$

i.e. $\sigma_{N}^{2}$ is infinitesimally close to $\sigma_{N_{0}}^{2}$ over the intermediate time-scale specified by $\left(N-N_{0}\right)<O\left(1 / \lambda_{N_{0}}^{2}\right)$ and can be said to have attained an asymptotic value. It follows then that the scalar distribution in the vicinity of $y \in W_{\beta}^{u}$, in the presence of small scalar diffusion, will not change by any appreciable amount over an intermediate timescale and can be said to have attained an asymptotic distribution. In fact, at larger perturbation amplitude $\beta$, the $\lambda_{n}$ are larger (see figure 23) and the asymptotic values, $\lambda_{N_{0}}$ and $\sigma_{N_{0}}^{2}$, are attained in shorter time, i.e. $N_{0}$ decreases with $\beta$. This can explain the numerically observed feature that the asymptotic scalar distribution is attained over a shorter time-scale at larger perturbation amplitudes (compare figure 4 with figure 6). Since the arguments hold for any cross-section $\bar{\phi}$ and any $y \in W_{\beta}^{u}(\bar{\phi})$, the scalar field in the entire bubble region will attain an asymptotic distribution. Moreover, at any particular point the asymptotic scalar value will be time-periodic and will have the same period as the period $T$ of the advection field. Numerically obtained time-series in figure 11(b) support this prediction.

\subsection{Effect of local expansion rates}

We consider next the effect of an increase in local expansion rates. Fix $y \in W_{\beta}^{u}$, let $y \equiv y^{N}, N>N_{1}$, and suppose $N_{1}\left(\epsilon_{1}, y\right)$ is unchanged while the $\beta_{n}$ are increased. Increasing $\beta_{n}$ results in larger $\lambda_{N}$, which means a 'thinner' zero-diffusivity distribution at $y$. Further, from (5.3), larger $\lambda_{n}$ yield smaller $\sigma_{N}^{2}$, and hence the scalar distribution at $y$, in the presence of scalar diffusion, is confined even closer to $W_{\beta}^{u}$. Therefore, as the dynamics becomes more strongly hyperbolic, the zero-diffusivity solution is wrapped more tightly around $W_{\beta}^{u}$ and diffusion is less effective as a smoothing process. Together, they account for the localized and non-uniform distributions obtained at larger perturbation amplitudes. Of course we have no method for increasing $\beta_{n}$ without changing $\beta$, and consequently changing the geometrical structure of $W_{\beta}^{u}$. We cannot then consider a fixed $y$ and examine the effect of increasing the $\beta_{n}$. The only alternative is to compare $y$, lying on $W_{\beta}^{u}$ corresponding to different $\beta$, that have approximately the same $N_{1}\left(\epsilon_{1}, y\right)$ for given $\epsilon_{1}$. To examine this situation, we choose three representative $y: y_{1} \in W_{\beta=0.2}^{u}, y_{2} \in W_{\beta=0.6}^{u}, y_{3} \in W_{\beta=0.8}^{u}$, all with the same $N_{1}\left(\epsilon_{1}, y\right)$ for $\epsilon_{1}$ small. We choose $y_{i} \equiv y_{i}^{N}$, with $N=10, i=1$, 2, 3, i.e. we are considering $t=10 T+t_{0}$. In figure 23 we plot $\beta_{n}, n \in[1,10]$, for all three cases; for given $y_{i}, \beta_{n}$ is the expansion 


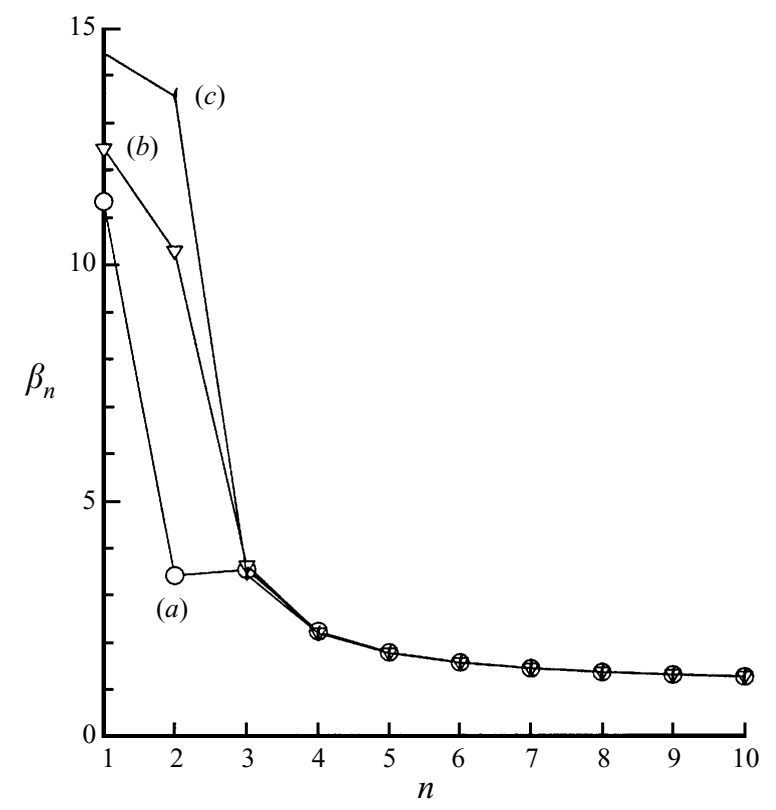

FIGURE 23. Local expansion rates, $\beta_{n}$, at points on the exact trajectory $\left\{y_{i}^{n}\right\}_{n=N}^{0}, N=10, i=1,2,3$, and $(a) y_{1}^{N}=(0.55,0.1649) \in W_{\beta=0.2}^{u},(b) y_{2}^{N}=(0.5,0.15288) \in W_{\beta=0.6}^{u},(c) y_{3}^{N}=(0.5,0.1476) \in W_{\beta=0.8}^{u}$. The perturbation frequency $\omega=0.72$ in all three cases. The points $y_{1}^{N}$ and $y_{2}^{N}$ are plotted in figures $2(b)$ and $(a)$ respectively.

factor of $f^{-1}$ in the local stable direction of $f^{-n+1}\left(y_{i}\right)$. Evidently, at larger $\beta$, the $\beta_{n}$ and consequently the $\lambda_{n}$ are larger on account of the increased hyperbolicity of the dynamics under the Poincare map $f \equiv P_{\beta}$. Not surprisingly the $\beta_{n}$ approach unity as $n$ increases and preimages of $y_{i}$ approach the non-hyperbolic separation point $p^{-}$.

Another method for increasing on average the local expansion rates in the chaotic bubble region is by modulating appropriately the coefficient $A_{122}$ in the timeindependent component $f^{u}(x)$ of the velocity field, given by (3.14). That this is indeed the case is shown by computing the local expansion rates or $\beta_{n}$ along the backward dynamical trajectory (under $f^{-1}$ ) through two representative points $y_{A}, y_{B}$ lying on the unstable manifolds $W_{A}, W_{B}$ respectively, where $W_{A} \equiv W_{\beta}^{u}$ for $\beta=0.5$, $\omega=0.5$ and $A_{122}=4.5 K x_{s}$ while $W_{B} \equiv W_{\beta}^{u}$ for $\beta=0.5, \omega=0.5$ and $A_{122}=2 K x_{s}$, and again $N_{1}\left(\epsilon_{1}, y_{A}\right) \simeq N_{1}\left(\epsilon_{1}, y_{B}\right)$. Note that the perturbation parameters $\beta, \omega$ are chosen to be identical to those in figures 14 and 15 . The numerical results, displayed in figure 24, show that the local expansion rates for $A_{122}=2 K x_{s}$ are significantly higher than that for $A_{122}=4.5 K x_{s}$. Lowering $A_{122}$ not only increases the size of the unperturbed bubble and consequently the size of the chaotic bubble region in the associated time-periodically perturbed flow, but also increases on average the local expansion rates. Then, following our discussion above, it is expected that the scalar distribution would become more non-uniform and localized about $W_{\beta}^{u}$ at lower $A_{122}$. This explains the observations made in $\S 3.6$ regarding the sharp qualitative difference between the scalar distributions of figures $14(a, b)$ and $15(a, b)$ corresponding to two different values of the coefficient $A_{122}$. We note that the perturbation parameters $\beta$, $\omega$ were chosen in a manner such that for both values of $A_{122}$ considered here, the corresponding Poincare maps have no KAM tori enclosing the core region of the bubble. In fact, the surviving KAM tori are of negligible size in both cases. Further, 


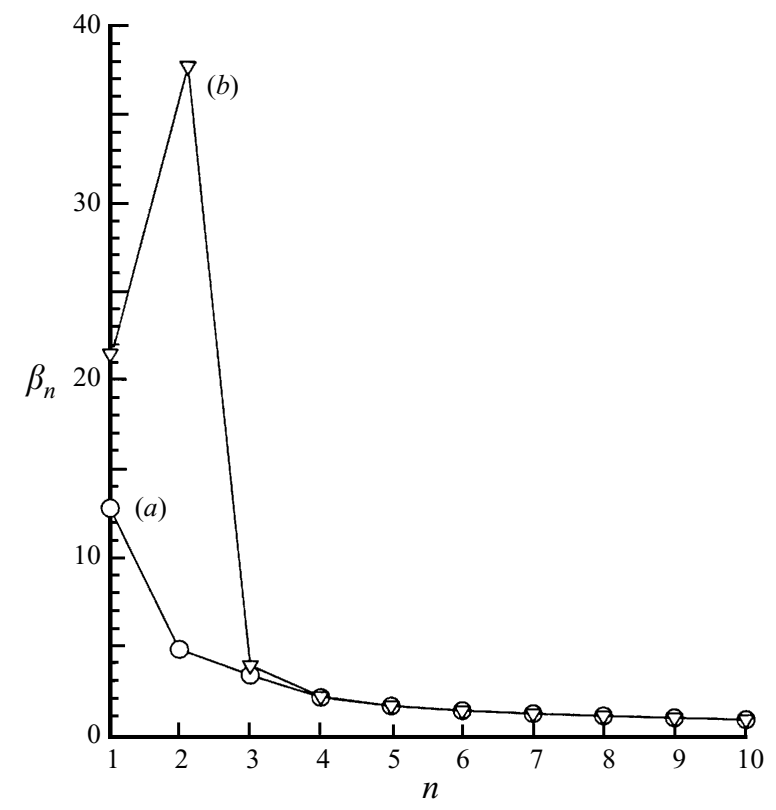

FiguRE 24. Local expansion rates, $\beta_{n}$, at points on the exact trajectory $\left\{y_{A, B}^{n}\right\}_{n=N}^{0}, N=10$, and (a) $y_{A}^{N}=(0.50352,0.11174) \in W_{A},(b) y_{B}^{N}=(-0.30030,0.58182) \in W_{B}$. Case $(a)$ corresponds to $A_{122}=4.5 K x_{s}$ and case $(b)$ corresponds to $A_{122}=2 K x_{s}$, where $A_{122}$ is the coefficient in (3.14). The perturbation parameters are $\beta=0.5, \omega=0.5$ in both cases.

the value of $\omega=0.5$ turns out to be close to the values of the perturbation frequency at which the amplitude $A(\omega)$ of the Melnikov's function (see $\S 4$ ) is maximum in either case; the value of $\omega$ at which $A(\omega)$ is maximum is of course different for different $A_{122}$, but are fortunately not widely separated for the two $A_{122}$ considered here.

\subsection{Distribution along the unstable manifold}

We consider finally how the distribution varies, at a fixed time $t=N T+t_{0}$, as one moves further away from $p^{-}$in terms of arclength along $W_{\beta}^{u}$. As $y \in W_{\beta}^{u}$ moves away from $p^{-}$in terms of arclength along $W_{\beta}^{u}, N_{1}\left(\epsilon_{1}, y\right)$ increases and the zerodiffusivity solution attains a non-zero value at $y$ at a later time. Consider two points $y_{1}, y_{2} \in W_{\beta}^{u}$, where $y_{1}<_{l} y_{2}$, i.e. $y_{1}$ is closer to $p^{-}$in terms of arclength along $W_{\beta}^{u}$. Fix $N>N_{1}\left(\epsilon_{1}, y_{2}\right)>N_{1}\left(\epsilon_{1}, y_{1}\right)$. Now, our numerical simulations show that the local expansion rates or $\beta_{n}$ in general decrease monotonically along each backward trajectory $\left\{y^{n}\right\}_{n=N}^{0}$ on $W_{\beta}^{u}$; this fact is evident from figure 23 . On a cautionary note we add that this may be violated if the backward trajectory through $y \in W_{\beta}^{u}$ passes through regions with homoclinic tangencies, since these regions are also characterized by weak stretching; figure 24 shows one such case where the monotonic decrease in $\beta_{n}$ is violated in one iterate. Since $f^{-n}\left(y_{1}\right)<_{l} f^{-n}\left(y_{2}\right)$ for all $n \in[0, N]$ and, as long as $\beta_{n}$ decreases monotonically as $p^{-}$is approached along $W_{\beta}^{u}, \lambda_{n}\left(y_{1}\right)<\lambda_{n}\left(y_{2}\right)$, where $\lambda_{n}\left(y_{i}\right)$ is the expansion factor of $f^{-n}$ along the stable direction of $y_{i}$. Therefore $\lambda_{N}\left(y_{1}\right)<\lambda_{N}\left(y_{2}\right)$, and the zero-diffusivity distribution at $y_{2}$ is thinner than at $y_{1}$. Further, because of the larger $\lambda_{n}\left(y_{2}\right), \sigma_{N}^{2}\left(y_{2}\right)<\sigma_{N}^{2}\left(y_{1}\right)$. Therefore a thinner zero-diffusivity distribution at $y_{2}$ is smoothed against a Gaussian with smaller variance, which means the scalar distribution about $W_{\beta}^{u}$, in the presence of scalar diffusion, should in general 
narrow down as one moves further away from $p^{-}$in terms of arclength along $W_{\beta}^{u}$; note that there is a (weak) gradient in the zero-diffusivity solution along $W_{\beta}^{u}$ (see figure $20 a$ ), and $\theta_{f r}\left(y_{2}, t=N T+t_{0}\right)<\theta_{f r}\left(y_{1}, t=N T+t_{0}\right)$. From figures $3(a-d)$ and $6(a-d)$ it is evident that the numerical simulation results agree with the predictions made above, with the exception of regions where the manifold $W_{\beta}^{u}$ has large curvature. Since these are also the regions where homoclinic tangencies are likely (Farmer \& Sidorowich 1991), the discrepancy is not entirely surprising. Note that since $W_{\beta}^{u}$ is not a submanifold of $\mathbb{R}^{2}$, segments of $W_{\beta}^{u}$ wind back infinitesimally close to each other such that the distributions along them may overlap, which can make verification of the previous prediction difficult (e.g. see figure 4). Also, for points $y \in W_{\beta}^{u}$ that are far away from $p^{-}$in terms of arclength along $W_{\beta}^{u}$, the backward trajectory through $y$ is bound to pass through weakly stretched regions as $W_{\beta}^{u}$ has a folded structure with several segments of large curvature signalling the likelihood of homoclinic tangencies, and for these points the theory becomes uncertain owing to likely violations in the assumption of monotonic decrease in $\beta_{n}$ along the backward trajectory $\left\{y^{n}\right\}_{n=N}^{0}$. The predictions are therefore more likely to hold for the situation in figures $3(a-d)$ and $6(a-d)$ where the scalar distribution remains localized along $W_{\beta}^{u}$ up to only a relatively short distance in terms of arclength along $p^{-}$as opposed to that in figure 4 . The arguments given above remain unchanged if $N_{1}\left(\epsilon_{1}, y\right)$ is replaced by $N_{0}\left(\epsilon_{0}, y\right)$, and therefore the conclusions are identical for the asymptotic scalar distribution. Moreover, since $N_{0}\left(\epsilon_{0}, y\right)$ increases as $y \in W_{\beta}^{u}$ moves away from $p^{-}$in terms of arclength along $W_{\beta}^{u}$, the asymptotic distribution is attained over a longer time at points further away from $p^{-}$in terms of arclength along $W_{\beta}^{u}$. Finally, as $N_{0}$ increases, the asymptotic value of the standard deviation of the Gaussian weighting function will become much greater than the asymptotic width of the zero-diffusivity solution about $W_{\beta}^{u}$, i.e. $\sigma_{N_{0}} \gg O\left(1 / \lambda_{N_{0}}\right)$, in which case a Gaussian smoothing of the zero-diffusivity solution will be nearly zero, and therefore the asymptotic scalar distribution, in the presence of small scalar diffusion, will tend to zero as one moves far away from $p^{-}$in terms of arclength along $W_{\beta}^{u}$. Comparing figure $2(a)$ with figure $3(d)$ shows that such is indeed the case.

We refrain from making any quantitative comparisons between scalar values obtained from our random-walk schemes and that predicted using the asymptotic shadowing result of (5.3). The main difficulty is the arbitrariness involved in choosing $t_{0}$, which is the cut-off time for the boundary layer solution of $\S 4$ and determines the 'initial' distribution for the fictitious zero-diffusivity solution. While the zero-diffusivity solution thus constructed offers a convenient heuristic tool for examining the role of small scalar diffusion, it seems difficult to extract any quantitative information from it. Moreover, due to the abundance of homoclinic tangencies in the near-wall region there is a lack of good (small $\delta$ ) deterministic shadowing (Ghosh 1994), and quantitative results based on (5.3) are likely to be unsatisfactory.

For the case with surviving KAM tori enclosing the core region of the bubble, the presence of invariant closed curves as well as the islands of stability enclosed within precludes the possibility of applying shadowing theory. In fact, the theory becomes uncertain whenever there are invariant curves since Wiener trajectories crossing invariant curves will not be shadowed (Klapper 1992a) and diffusion can produce effects that will not be captured by the shadowing theory. For the cases considered in this section, the flow-domain has no invariant curves and is very much the basis for arguing that shadowing theory will continue to give the correct qualitative results even when small $\delta$ shadowing is unlikely. 


\section{Conclusions}

We have examined scalar advection-diffusion in a two-dimensional flow with chaotic particle trajectories adjacent to an active no-slip surface at the limit of large Péclet number or small scalar diffusivity using results from dynamical systems theory. The flow field was constructed in the form of a Taylor series expansion satisfying the no-slip boundary condition and consistent with the Navier-Stokes equations. An unstable manifold emanating from a point of zero time-averaged shear stress on the no-slip boundary is found to play a dominant role in the advection-diffusion of passive scalar from the boundary, particularly when the underlying chaotic particle motion is widespread and the dynamics in the flow has a pronounced hyperbolic character. It is found that the increased hyperbolicity results in scalar distributions that are localized entirely along the unstable manifold with sharp scalar gradients. Introducing a fictitious zero-diffusivity solution provides an understanding of the relationship between the stirring and mixing processes and shadowing theory gives a nice physical description of the role of small scalar diffusion as a local smoothing of fine scale variations created by the stirring process. A novel feature in this problem is the presence of the no-slip boundary and the interaction between the non-hyperbolicity at the boundary and the strongly hyperbolic character of the overlying chaotic advection in the bubble-region of the flow. The non-hyperbolicity at the boundary is found to play an important role in the advection-diffusion process and strongly influences the time-evolution of the scalar field. The wall-integrated flux remains $O\left(P e^{-2 / 3}\right)$ even in the chaotic-advection case. Introducing a time-periodic perturbation in the steady advection field, again with low-order polynomial behaviour in space, introduces only weak time and space dependence in the near-wall thermal boundary layer after the initial transients have died down, resulting in weak enhancement in the asymptotic flux over the steady-advection case. The absence of near-wall structures that have strong temporal and spatial fluctuations parallel to the wall is the likely cause for the observed weak time-dependence in the thermal boundary layer, and the consequent lack of enhanced transport usually associated with turbulent flows. Our numerical simulation results show that in the presence of widespread chaotic particle motion the scalar distribution attains an asymptotic distribution over a time-scale that is much shorter than the time-scale over which an asymptotic distribution is attained in the steady flow. Moreover, this asymptotic distribution is found to be time-periodic with the same period as that of the chaotic advection field.

We would like to acknowledge the parallel computing facilities made generously available by the Center for Advanced Computing Research at Caltech. This research was supported by a grant from the Air Force Office of Scientific Research, Grant No. AFOSR-91-0241 and by a grant from the Office of Naval Research, Grant No. N00014-97-1-0071.

\section{REFERENCES}

Acrivos, A. \& Goddard, J. D. 1965 Asymptotic expansions for laminar foced-convection heat and mass transfer. Part 1. Low speed flows J. Fluid Mech. 23, 273.

Anosov, D. V. 1967 Geodesic flows on closed Riemannian manifolds with negative curvature. Proc. Steklov Inst. Math. 90, 1 (Am. Math. Soc. translation 1969).

Aref, H. 1984 Stirring by chaotic advection. J. Fluid Mech. 143, 1.

Aref, H. \& Jones, S. W. 1989 Enhanced separation of diffusing particles by chaotic advection. Phys. Fluids A 1, 470.

ARnolD, L. 1974 Stochastic Differential Equations. Wiley. 
BAtChelor, G. K. 1956 Steady laminar flow with closed streamlines at large Reynolds number. J. Fluid Mech. 1, 177.

Bowen, R. $1975 \omega$-limit sets for Axiom A diffeomorphisms. J. Diff Equat. 18, 333.

Camassa, R. \& Wiggins, S. 1991 Chaotic advection in a Rayleigh-Bénard flow. Phys. Rev. A 43, 774.

Chandrasekhar, S. 1943 Stochastic problems in Physics and Astronomy. Rev. Mod. Phys. 15, 1.

Chorin, A. J. 1973 Numerical study of slightly viscous flow. J. Fluid Mech. 57, 785.

Chorin, A. J. \& Marsden, J. E. 1979 A Mathematical Introduction to Fluid Mechanics. Springer.

Dallmann, U. 1988 Three-dimensional vortex structures and vorticity topology. Fluid Dyn. Res. 3, 183.

Easton, R. W. 1986 Trellises formed by stable and unstable manifolds in the plane. Trans. Am. Math. Soc. 294(2), 719.

ECKART, C. 1948 An analysis of the stirring and mixing processes in incompressible fluids. J. Mar. Res. 7, 265.

Farmer, J. D. \& Sidorowich, J. J. 1991 Optimal shadowing and noise reduction. Physica D 47, 373.

GHosh, S. 1994 The role of various geometrical structures in scalar advection-diffusion. PhD thesis, California Institute of Technology.

Ghosh, S., Chang, H.-C. \& Sen, M. 1992 Heat-transfer enhancement due to slender recirculation and chaotic transport between counter-rotating eccentric cylinders. J. Fluid Mech. 238, 119.

Grebogi, C., Hammel, S. M., Yorke, J. A. \& Sauer, T. 1990 Shadowing of physical trajectories in chaotic dynamics: containment and refinement. Phys. Rev. Lett. 65, 1527.

Haji-Sheikh, A. \& Sparrow, E. M. 1967 The solution of heat conduction problems by probability methods. Trans ASME C J. Heat Transfer 89, 121.

Hammel, S. M. 1990 A noise reduction method for chaotic systems. Phys. Lett. A 148, 421.

Hammel, S. M., Yorke, J. A. \& Grebogi, C. 1987 Do numerical orbits of chaotic dynamical processes represent true orbits? J. Complexity 3, 136.

Hammel, S. M., Yorke, J. A. \& Grebogi, C. 1988 Numerical orbits of chaotic processes represent true orbits. Bull. Am. Math. Soc. 19, 465.

Hirsch, M. W., Pugh, C. C. \& Shub,M. 1977 Invariant Manifolds. Springer Lecture Notes in Mathematics, Vol. 583. Springer.

JONES, S. W. 1991 The enhancement of mixing by chaotic advection. Phys. Fluids A 3, 1081.

Khakhar, D. V. \& Ottino, J. M. 1986 Fluid mixing (stretching) by time-periodic sequences of weak flows. Phys. Fluids 29, 3503.

KLAPPER, I. $1992 a$ Shadowing and the role of small diffusivity in the chaotic advection of scalars. Phys. Fluids A 4, 861.

KLAPPER, I. 1992 b A study of fast dynamo action in chaotic helical cells. J. Fluid Mech. 239, 359.

McKean, H. P. 1969 Stochastic Integrals. Academic.

Molchanov, S. A., Ruzmaikin, A. A. \& Sokolov, D. D. 1985 Kinematic dynamo action in random flow. Sov. Phys. Usp. 28, 307.

Pan, F. Y. \& Acrivos, A. 1968 Heat transfer at high Péclet number in regions of closed streamlines. Intl J. Heat Mass Transfer 11, 439.

PedLeY, T. J. 1972 On the forced heat transfer from a hot film embedded in the wall in twodimensional unsteady flow. J. Fluid Mech. 55, 329.

Perry, A. E. \& ChONg, M. S. 1986 a A series-expansion study of the Navier-Stokes equations with applications to three-dimensional separation patterns. J. Fluid Mech. 173, 203.

Perry, A. E. \& ChOng, M. S. $1986 b$ A series-expansion study of the Navier-Stokes equations with applications to three-dimensional separation patterns - a detailed treatment. Rep. FM-17. Mech. Engng Dept., University of Melbourne.

Press, W. H., Flannery, B. P., Teukolsky, S. A. \& Vetterling, W. T. 1986 Numerical Recipes. Cambridge University Press.

Rhines, P. B. \& Young, W. R. 1983 How rapidly is a passive scalar mixed within closed streamlines? J. Fluid Mech. 133, 133.

Rom-Kedar, V., Leonard, A. \& Wiggins, S. 1990 An analytical study of transport, mixing, and chaos in an unsteady vortical flow. J. Fluid Mech. 214, 347.

Saffman, P. G. 1959 A theory of dispersion in porous medium. J. Fluid Mech. 6, 321. 
Shariff, K., Pulliam, T. H. \& Ottino, J. M. 1991 A dynamical systems analysis of kinematics in the time-periodic wake of a circular cylinder. Lectures Appl. Math. 28, 613.

Smale, S. 1967 Differentiable dynamical systems. Bull. Am. Math. Soc. 73, 747.

Toвak, M. \& Peake, D. J. 1982 Topology of three-dimensional separated flows. Ann. Rev. Fluid Mech. 14, 61.

Wang, M. C. \& Uhlenbeck, G. E. 1945 On the theory of the Brownian motion II. Rev. Mod. Phys. $17,323$.

Wiggins, S. 1990 Introduction to Applied Nonlinear Dynamical Systems and Chaos. Springer.

WigGins, S. 1992 Chaotic Transport in Dynamical Systems. Springer. 